\title{
OPTIMAL CONTROL OF SEMILINEAR ELLIPTIC EQUATIONS IN MEASURE SPACES*
}

\author{
EDUARDO $\mathrm{CASAS}^{\dagger}$ AND KARL KUNISCH ${ }^{\ddagger}$
}

\begin{abstract}
Optimal control problems in measure spaces governed by semilinear elliptic equations are considered. First order optimality conditions are derived and structural properties of their solutions, in particular sparsity, are discussed. Necessary and sufficient second order optimality conditions are obtained as well. On the basis of the sufficient conditions, stability of the solutions is analyzed. Highly nonlinear terms can be incorporated by utilizing an $L^{\infty}(\Omega)$ regularity result for solutions of the first order necessary optimality conditions.
\end{abstract}

Key words. measure controls, optimal control, sparsity, semilinear elliptic equation, first and second order optimality conditions, stability analysis

AMS subject classifications. 90C48, 49J52, 49K20, 35J61

DOI. $10.1137 / 13092188 \mathrm{X}$

1. Introduction. This paper is dedicated to the study of the optimal control problem

$$
\min _{u \in \mathcal{M}(\omega)} J(u)=\frac{1}{2}\left\|y-y_{d}\right\|_{L^{2}(\Omega)}^{2}+\alpha\|u\|_{\mathcal{M}(\omega)},
$$

where $y$ is the unique solution to the Dirichlet problem

$$
\left\{\begin{aligned}
-\Delta y+a(x, y)=u & \text { in } \Omega \\
y=0 & \text { on } \Gamma .
\end{aligned}\right.
$$

The control domain $\omega$ is a relatively closed subset of $\Omega$. We assume that $\alpha>0$, $y_{d} \in L^{2}(\Omega)$, and $\Omega$ is a bounded domain in $\mathbb{R}^{n}, n=2$ or 3 , with Lipschitz boundary $\Gamma$. The controls are taken in the space of regular Borel measures $\mathcal{M}(\omega)$. As usual, $\mathcal{M}(\omega)$ is identified by the Riesz theorem with the dual space of $C_{0}(\omega)$-consisting of the continuous functions in $\bar{\omega}$ vanishing on $\Gamma \cap \bar{\omega}$ - endowed with the norm

$$
\|u\|_{\mathcal{M}(\omega)}=\sup _{\|z\|_{C_{0}(\omega)} \leq 1}\langle u, z\rangle=\sup _{\|z\|_{C_{0}(\omega)} \leq 1} \int_{\omega} z d u
$$

which is equivalent to the total variation of $u$; see Rudin [19].

We recall that the use of measure-valued controls is motivated by their sparsity promoting properties. If $u \in L^{1}(\omega)$, then $\|u\|_{\mathcal{M}(\omega)}$ and $\int_{\Omega}|u| d x$ coincide. However, the consideration of $(\mathrm{P})$ in $L^{1}(\omega)$ does not allow us to argue existence of a minimizer,

\footnotetext{
* Received by the editors May 22, 2013; accepted for publication (in revised form) November 8, 2013; published electronically February 4, 2014.

http://www.siam.org/journals/sicon/52-1/92188.html

†Departmento de Matemática Aplicada y Ciencias de la Computación, E.T.S.I. Industriales y de Telecomunicación, Universidad de Cantabria, 39005 Santander, Spain (eduardo.casas@unican.es). This author's research was supported by Spanish Ministerio de Economía y Competitividad under project MTM2011-22711.

${ }^{\ddagger}$ Institute for Mathematics and Scientific Computing, University of Graz, Heinrichstrasse 36, A-8010 Graz, Austria (karl.kunisch@uni-graz.at). This author's research was supported by the Austrian Science Fund (FWF) under grant SFB F32 (SFB "Mathematical Optimization and Applications in Biomedical Sciences").
} 
whereas the larger space $\mathcal{M}(\omega)$ does. The choice of $\mathcal{M}(\omega)$ in the cost functional is also useful for the optimal actuator placement. Moreover the cost of the control enters (P) in a manner that is linearly proportional rather than the frequently investigated quadratic costs.

Sparsity promoting controls were investigated in several earlier works. Some of them consider the case of measure-valued controls as done here (see $[7,8,12,14]$ ); others use additionally pointwise control constraints. In this case the $\mathcal{M}(\omega)$ norm can be equivalently replaced by the $L^{1}(\omega)$ norm; see $[16,18,20,23]$. In the previous papers, the state equation is linear. The case of semilinear elliptic equations with $L^{\infty}(\Omega)$ controls controls is studied in $[9,10,11]$.

The paper is organized as follows. In section 2 we provide the necessary analysis of the state equation, including differentiability of the state with respect to the control. Necessary first and second order optimality conditions are derived in section 3 . A second order sufficient optimality condition is achieved in section 4. This condition allows a stability analysis of the solutions to perturbations in $y_{d}$ and possible perturbations on the right-hand side of the equation. In the case that $\omega=\Omega$ extra regularity of controls and states which satisfy the first order necessary condition can be obtained. This is carried out in section 5 . The $L^{\infty}(\Omega)$ bound of these states can be used to allow for highly nonlinear terms in $a(x, y)$. This is exploited in section 6 .

2. Analysis of the state equation. In this section, we will establish the existence and uniqueness of the solution of the state equation (1.2) as well as the continuity and differentiability properties of the control-to-state mapping. For the well-posedness we will use the following assumption.

(A1) The mapping $a: \Omega \times \mathbb{R} \rightarrow \mathbb{R}$ is a Carathéodory function, nondecreasing monotone with respect to the second variable for almost every $x \in \Omega$, and satisfying for almost all $x \in \Omega$ and all $s \in \mathbb{R}$

$$
\left\{\begin{array}{l}
\exists C_{a}>0 \text { and } \exists \phi_{0} \in L^{1}(\Omega) \text { such that } \\
|a(x, s)| \leq\left|\phi_{0}(x)\right|+C_{a}|s|^{r} \quad \text { with } r<\left\{\begin{array}{cc}
+\infty & \text { if } n=2, \\
3 & \text { if } n=3 .
\end{array}\right.
\end{array}\right.
$$

We say that $y \in L^{1}(\Omega)$ is a solution to $(1.2)$ if $a(\cdot, y) \in L^{1}(\Omega)$ and

$$
\int_{\Omega}(-y \Delta z+a(x, y) z) d x=\int_{\omega} z d u \quad \forall z \in \mathcal{Z}
$$

where

$$
\mathcal{Z}=\left\{z \in H_{0}^{1}(\Omega): \Delta z \in C(\bar{\Omega})\right\} .
$$

Observe that $\mathcal{Z} \subset C_{0}(\Omega)$. Thus, all the integrals in (2.2) are well defined.

THEOREM 2.1. Under assumption (A1), there exists a unique solution y of (1.2). Moreover, it satisfies that $y \in W_{0}^{1, p}(\Omega)$ for every $p<n /(n-1)$ and

$$
\|y\|_{W_{0}^{1, p}(\Omega)} \leq C_{p}\left(\|a(\cdot, 0)\|_{L^{1}(\Omega)}+\|u\|_{\mathcal{M}(\omega)}\right)
$$

for some constant $C_{p}$ independent of $u \in \mathcal{M}(\omega)$. Finally, if $u_{k} \stackrel{*}{\rightarrow} u$ in $\mathcal{M}(\omega)$, then $y\left(u_{k}\right) \rightarrow y(u)$ strongly in $W_{0}^{1, p}(\Omega)$ for every $1 \leq p<n /(n-1)$.

This result was first proved by Brezis and Strauss [6] for functions $u \in L^{1}(\Omega)$ without the growth assumption given in (2.1). Later, Benilan and Brezis [2] observed 
that the situation for measures is different. Specifically, they showed that (1.2) has no solution for $a(x, s)=s^{3}, n=3$, and $u=\delta_{x_{0}}$, where $x_{0}$ is a point in $\Omega$. A way to ensure the existence of a solution for problem (1.2) consists of assuming the growth condition on $a$ expressed in (2.1); see Boccardo and Gallouët [3]. For the sake of completeness, let us give an independent proof of the existence of a solution that illustrates the difficulty of passing from $L^{1}$ functions to measures and the role played by the growth condition (2.1).

Proof. We first consider

$$
\left\{\begin{aligned}
-\Delta \zeta=u & \text { in } \Omega \\
\zeta=0 & \text { on } \Gamma
\end{aligned}\right.
$$

It is well known (see, e.g., [21]) that (2.4) admits a unique solution $\zeta \in W_{0}^{1, p}(\Omega)$ for every $1 \leq p<\frac{n}{n-1}$ and

$$
\|\zeta\|_{W_{0}^{1, p}(\Omega)} \leq c_{p}\|u\|_{\mathcal{M}(\omega)}
$$

for a constant $c_{p}$ independent of $u$. Note that $\zeta \in W_{0}^{1, p}(\Omega)$ implies that $\zeta \in L^{p^{*}}(\Omega)$ for any $1 \leq p^{*}<+\infty$ if $n=2$ and any $1 \leq p^{*}<3$ if $n=3$.

Let us further consider

$$
\left\{\begin{aligned}
-\Delta w+g(x, w) & =f(x) & & \text { in } \Omega, \\
w & =0 & & \text { on } \Gamma,
\end{aligned}\right.
$$

where

$$
g(x, s)=a(x, \zeta(x)+s)-a(x, \zeta(x)), \quad f(x)=-a(x, \zeta(x)) .
$$

From the growth condition assumed in (A1), we have that $f \in L^{1}(\Omega)$ and

$$
\sup \{|g(x, s)|:|s| \leq t\} \leq \phi_{0}(x)+C_{a}|t|^{r} \in L^{1}(\Omega) \quad \forall t>0 .
$$

Moreover, the monotonicity of $a$ with respect to the second variable implies $g(x, s) s \geq 0$ for all $s \in \mathbb{R}$. With these properties the existence of a solution $w \in$ $W_{0}^{1,1}(\Omega)$ of (2.6) follows; see [3, Theorem 2]. Setting $y=w+\zeta \in W_{0}^{1,1}(\Omega)$ gives a solution to (1.2). By [5, Corollary B1] this solution is unique.

To verify the a priori estimate, we express (1.2) in the form

$$
\left\{\begin{aligned}
-\Delta y+h(x, y) & =u-a(x, 0) & & \text { in } \Omega, \\
y & =0 & & \text { on } \Gamma,
\end{aligned}\right.
$$

where $h(x, s)=a(x, s)-a(x, 0)$. From the proof of [3, Theorem 3] it follows that

$$
\|h(\cdot, y)\|_{L^{1}(\Omega)} \leq\|u-a(\cdot, 0)\|_{\mathcal{M}(\Omega)} \leq\|u\|_{\mathcal{M}(\omega)}+\|a(\cdot, 0)\|_{L^{1}(\Omega)} .
$$

Using again [21], as in (2.5), we deduce that $y \in W_{0}^{1, p}(\Omega)$ for every $1 \leq p<n /(n-1)$ and

$$
\|y\|_{W_{0}^{1, p}(\Omega)} \leq c_{p}\|u-a(\cdot, y)\|_{\mathcal{M}(\Omega)} \leq c_{p}\left(\|u\|_{\mathcal{M}(\omega)}+2\|a(\cdot, 0)\|_{L^{1}(\Omega)}\right),
$$

which implies the estimate (2.3).

Finally, let us prove the claimed continuity. From (2.3) we get the existence of a subsequence, denoted in the same way, $y_{k}=y\left(u_{k}\right) \rightarrow y$ in $W_{0}^{1, p}(\Omega)$ for all 
$1 \leq p<n /(n-1)$. Hence, $y_{k} \rightarrow y$ strongly in $L^{p^{*}}(\Omega)$. By the Lebesgue dominated convergence theorem and (A1) we obtain that $a\left(\cdot, y_{k}\right) \rightarrow a(\cdot, y)$ strongly in $L^{1}(\Omega)$. Now, we can pass to the limit in the equations satisfied by $u_{k}$ and $y_{k}$ and deduce that $y$ is the state associated to $u$. By uniqueness of the solution of the state equations we conclude that the whole sequence $\left\{y_{k}\right\}_{k=1}^{\infty}$ converges weakly to $y$ in $W_{0}^{1, p}(\Omega)$. Now, from the compact embeddings of $\mathcal{M}(\omega)$ and $L^{1}(\Omega)$ in $W^{-1, p}(\Omega)$ for all $1 \leq$ $p<n /(n-1)$, we get that $u_{k}-a\left(\cdot, y_{k}\right) \rightarrow u-a(\cdot, y)$ strongly in $W^{-1, p}(\Omega)$ for the mentioned range of $p$. Applying the result by Jerison and Kenig [17, Theorem 0.5], we conclude the strong convergence of $\left\{y_{k}\right\}_{k=1}^{\infty}$ to $y$ in the space $W_{0}^{1, p}(\Omega)$.

Now, we define the space

$$
V(\Omega)=\left\{y \in W_{0}^{1,1}(\Omega): \Delta y \in \mathcal{M}(\Omega)\right\},
$$

which is a Banach space when endowed with the norm

$$
\|y\|_{V(\Omega)}=\|y\|_{W_{0}^{1,1}(\Omega)}+\|\Delta y\|_{\mathcal{M}(\Omega)} .
$$

At this point we remark that $\mathcal{M}(\omega)$ is identified with a subspace of $\mathcal{M}(\Omega)$. We also observe that by $(2.5)$ the space $V(\Omega)$ is continuously included in $W_{0}^{1, p}(\Omega)$ for every $1 \leq p<n /(n-1)$.

In the remainder of this section we study the differentiability of the mapping $G: \mathcal{M}(\omega) \rightarrow V(\Omega)$ given by $G(u)=y(u)$ with $y(u)$ the solution of (1.2). To this end we make the following assumptions.

(A2) The mapping $a: \Omega \times \mathbb{R} \rightarrow \mathbb{R}$ is a Carathéodory function of class $C^{1}$ with respect to the second variable for almost all $x \in \Omega$, and it satisfies for almost all $x \in \Omega$ and all $s \in \mathbb{R}$

$$
\left\{\begin{array}{l}
\exists C_{a}>0 \text { and } \exists \phi_{1} \in L^{q_{1}}(\Omega) \text { with } q_{1}>\frac{n}{2} \text { such that } \\
0 \leq \partial_{y} a(x, s) \leq\left|\phi_{1}(x)\right|+C_{a}|s|^{r} \quad \text { with } r<\left\{\begin{array}{cc}
+\infty & \text { if } n=2, \\
2 & \text { if } n=3 .
\end{array}\right.
\end{array}\right.
$$

(A3) The mapping $a$ is a Carathéodory function of class $C^{2}$ with respect to the second variable for almost all $x \in \Omega$, and it satisfies for almost all $x \in \Omega$ and all $s \in \mathbb{R}$

$$
\left\{\begin{array}{l}
\exists C_{a}>0 \text { and } \exists \phi_{2} \in L^{q_{2}}(\Omega) \text { with } q_{2}>\left\{\begin{array}{ll}
1 & \text { if } n=2, \\
3 & \text { if } n=3
\end{array}\right. \text { such that } \\
\left|\partial_{y}^{2} a(x, s)\right| \leq\left|\phi_{2}(x)\right|+C_{a}|s|^{r} \quad \text { with } r<\left\{\begin{array}{cc}
+\infty & \text { if } n=2, \\
1 & \text { if } n=3 .
\end{array}\right.
\end{array}\right.
$$

We observe that if (A2) holds and $a(\cdot, 0) \in L^{1}(\Omega)$, then (A1) is satisfied. Similarly, if (A3) holds, $\partial_{y} a(\cdot, 0) \in L^{q_{1}}(\Omega)$ for some $q_{1}>\frac{n}{2}$, and $\partial_{y} a(x, s) \geq 0$, then (A2) also holds.

THEOREM 2.2. Under assumptions (A1) and (A2), $G: \mathcal{M}(\omega) \rightarrow V(\Omega)$ is of class $C^{1}$ and the derivative $z_{v}=G^{\prime}(u) v$ is the solution to

$$
\left\{\begin{aligned}
-\Delta z+\frac{\partial a}{\partial y}(x, y) z & =v & & \text { in } \Omega, \\
z & =0 & & \text { on } \Gamma
\end{aligned}\right.
$$

where $y=G(u)$. Furthermore, if (A3) holds, then $G$ is of class $C^{2}$ and $w=$ $G^{\prime \prime}(u)\left(v_{1}, v_{2}\right)$ is the solution to

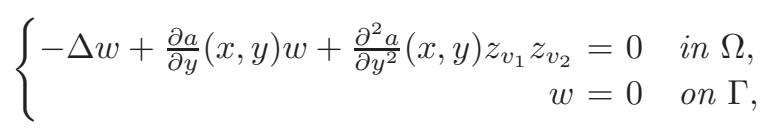

where $z_{v_{i}}=G^{\prime}(u) v_{i}, i=1,2$. 
Proof. Let us introduce the operator $\mathcal{F}: V(\Omega) \times \mathcal{M}(\omega) \rightarrow \mathcal{M}(\Omega)$ by

$$
\mathcal{F}(y, u)=-\Delta y+a(\cdot, y)-u .
$$

From (A2) and recalling that $V(\Omega) \subset W_{0}^{1, p}(\Omega)$ continuously, it follows that the mapping $y \rightarrow a(\cdot, y)$ is $C^{1}$ from $V(\Omega)$ to $L^{1}(\Omega) \subset \mathcal{M}(\Omega)$; see, for instance, [22, section 4.3]. Hence, $\mathcal{F}$ is a $C^{1}$ operator and $\partial_{y} \mathcal{F}(y, u): V(\Omega) \rightarrow \mathcal{M}(\Omega)$ is defined by

$$
\frac{\partial \mathcal{F}}{\partial y}(y, u) z=-\Delta z+\frac{\partial a}{\partial y}(x, y) z .
$$

Assumption (A2) implies that $\partial_{y} a(\cdot, y) \in L^{\rho}(\Omega)$ for some $\rho>n / 2$. Therefore, we can use [21, Theorem 9.1] to deduce that $\partial_{y} \mathcal{F}(y, u): V(\Omega) \rightarrow \mathcal{M}(\Omega)$ is an isomorphism. As an immediate consequence of the implicit function theorem we deduce that $G$ is a $C^{1}$ mapping and $G^{\prime}(u) v$ is given by (2.9).

Finally, if (A3) holds, then the mapping $y \rightarrow a(\cdot, y)$ is $C^{2}$, and consequently $\mathcal{F}$ is also $C^{2}$. Let us observe that (A3) and the fact that $y, z_{v_{1}}, z_{v_{2}} \in V(\Omega)$ imply that $\frac{\partial^{2} a}{\partial y^{2}}(x, y) z_{v_{1}} z_{v_{2}} \in L^{1}(\Omega)$. Once again the implicit function theorem implies that $G$ is of class $C^{2}$ and (2.10) is satisfied.

Remark 2.3. Due to the $W_{0}^{1, p}(\Omega)$ regularity of the solution $y$ to (1.2), we can integrate by parts in $(2.2)$ and use density of $C_{0}^{\infty}(\Omega)$ in $W_{0}^{1, p^{\prime}}(\Omega)\left(p^{\prime}<+\infty\right)$ to obtain

$$
\int_{\Omega}(\nabla y \nabla z+a(x, y) z) d x=\int_{\omega} z d u \quad \forall z \in W_{0}^{1, p^{\prime}}(\Omega) \quad \forall p^{\prime}>n .
$$

The same variational formulation is valid for (2.9) and (2.10).

3. Necessary optimality conditions for (P). From Theorem 2.1 the existence of a global minimum for problem $(\mathrm{P})$ is immediate. Since this problem is not convex, we are going to deal with local minimizers. Hereafter $\bar{u}$ will denote a local minimum of $(\mathrm{P})$ with associated state $\bar{y}$. Before stating the optimality conditions satisfied by $(\bar{y}, \bar{u})$, we analyze the differentiability of the cost functional. Let us express the cost in the form $J(u)=F(u)+\alpha j(u)$, where

$$
F(u)=\frac{1}{2}\left\|G(u)-y_{d}\right\|_{L^{2}(\Omega)}^{2} \quad \text { and } \quad j(u)=\|u\|_{\mathcal{M}(\omega)} .
$$

Proposition 3.1. Under assumptions (A1) and (A2), $F: \mathcal{M}(\omega) \rightarrow \mathbb{R}$ is of class $C^{1}$ and for all $u, v \in \mathcal{M}(\omega)$ we have

$$
F^{\prime}(u) v=\int_{\Omega}\left(y-y_{d}\right) z_{v} d x=\int_{\omega} \varphi d v
$$

where $y=G(u), z_{v}=G^{\prime}(u) v$, and $\varphi \in W_{0}^{1, p^{\prime}}(\Omega)$, for some $p^{\prime}>n$, is the solution to

$$
\left\{\begin{aligned}
-\Delta \varphi+\frac{\partial a}{\partial y}(x, y) \varphi & =y-y_{d} & & \text { in } \Omega, \\
\varphi & =0 & & \text { on } \Gamma .
\end{aligned}\right.
$$

If in addition (A3) holds, then $F$ is of class $C^{2}$, and for every $u, v_{1}, v_{2} \in \mathcal{M}(\omega)$ we have

$$
F^{\prime \prime}(u)\left(v_{1}, v_{2}\right)=\int_{\Omega}\left[\left(y-y_{d}\right) w+z_{v_{1}} z_{v_{2}}\right] d x=\int_{\Omega}\left[1-\varphi \frac{\partial^{2} a}{\partial y^{2}}(x, y)\right] z_{v_{1}} z_{v_{2}} d x
$$

where $w=G^{\prime \prime}(u)\left(v_{1}, v_{2}\right) \in V(\Omega)$, and $z_{v_{i}}=G^{\prime}(u) v_{i}$ for $i=1,2$. 
Proof. By using the chain rule and Theorem 2.1 the differentiability properties of $F$ and the first identities in (3.1) and (3.3) are obvious. Let us prove the $W_{0}^{1, p^{\prime}}(\Omega)$ regularity of $\varphi$ and the second identities of (3.1) and (3.3). We argue in dimension $n=3$ because in dimension $n=2$ the arguments are the same, but the fact that $V(\Omega) \subset L^{q}(\Omega)$ for all $q<+\infty$ makes the computations easier. First, we observe that $y \in V(\Omega) \subset L^{q}(\Omega)$ for all $1 \leq q<3$. Then, (A2) implies that $\partial_{y} a(\cdot, y) \in L^{q}(\Omega)$ for some $q>3 / 2$. Hence, applying Stampacchia estimates [21, Theorem 4.2] to (3.2) we deduce that $\varphi \in L^{\infty}(\Omega)$. Therefore, we have that $\Delta \varphi \in L^{q}(\Omega) \subset W^{-1, \hat{q}}(\Omega)$ for some $q>3 / 2$ and $\hat{q}>3$. Using again [17] we deduce that $\varphi \in W_{0}^{1, p^{\prime}}(\Omega)$ for some $3<p^{\prime} \leq \hat{q}$.

To prove the second identity of (3.1) it is enough to take into account Remark 2.3 and the regularity of $\varphi$, along with (2.9) and (3.2). The same argument is used to deduce the second identity of (3.3).

Concerning the functional $j: \mathcal{M}(\omega) \rightarrow \mathbb{R}, j(u)=\|u\|_{\mathcal{M}(\omega)}$, we note that it is Lipschitz continuous and convex. Hence, it has a subdifferential and a directional derivative, which are denoted by $\partial j(u)$ and $j^{\prime}(u ; v)$, respectively. The following propositions give some properties of $\partial j(u)$ and provide an expression for $j^{\prime}(u ; v)$.

Proposition 3.2. Let us assume that $\lambda \in \partial j(u)$ and $\lambda \in C_{0}(\omega)$; then we have $\|\lambda\|_{C_{0}(\Omega)} \leq 1$. Moreover, if $u \neq 0$, the following properties hold:

1. $\|\lambda\|_{C_{0}(\omega)}=1$ and $\int_{\omega} \lambda d u=\|u\|_{\mathcal{M}(\omega)}$.

2. Taking the Jordan decomposition $u=u^{+}-u^{-}$, we have

$$
\begin{aligned}
& \operatorname{supp}\left(u^{+}\right) \subset\{x \in \omega: \lambda(x)=+1\}, \\
& \operatorname{supp}\left(u^{-}\right) \subset\{x \in \omega: \lambda(x)=-1\} .
\end{aligned}
$$

The inequality $\|\lambda\|_{C_{0}(\Omega)} \leq 1$ follows easily from the definition of subdifferential. The reader is referred to [7] for the proof of 1 and to [7, Lemma 3.4] for 2.

Before considering the directional derivative $j^{\prime}(u ; v)$, let us introduce some notation. Given two measures $u, v \in \mathcal{M}(\omega)$, we consider the Lebesgue decomposition of $v=v_{a}+v_{s}$ with respect to $|u|$, where $v_{a}$ is the absolutely continuous part of $v$ with respect to $|u|$, and $v_{s}$ is the singular part. Now, we take the Radon-Nikodym derivative of $v_{a}$ with respect to $|u|, d v_{a}=g_{v} d|u|$. Then we have

$$
\|v\|_{\mathcal{M}(\omega)}=\left\|v_{a}\right\|_{\mathcal{M}(\omega)}+\left\|v_{s}\right\|_{\mathcal{M}(\omega)}=\int_{\omega}\left|g_{v}\right| d|u|+\left\|v_{s}\right\|_{\mathcal{M}(\omega)}
$$

In particular, it is obvious that $u$ is absolutely continuous with respect to $|u|$. Moreover we can express $d u=h d|u|$, where $h$ is measurable with respect to $|u|$ and $|h(x)|=1$ for all $x \in \omega, d u^{+}=h^{+} d|u|$, and $d u^{-}=h^{-} d|u|$, where $u=u^{+}-u^{-}$is the Jordan decomposition of $u$. See, for instance, [19, Chapter 6] for details.

Proposition 3.3. Let $u, v \in \mathcal{M}(\omega)$; then

$$
j^{\prime}(u ; v)=\int_{\omega} g_{v} d u+\left\|v_{s}\right\|_{\mathcal{M}(\omega)} .
$$

Proof. As above, let us write $d u=h d|u|$. Then we have

$$
\begin{aligned}
& \operatorname{supp}\left(u^{+}\right) \subset \omega_{+}=\{x \in \omega: h(x)=+1\}, \\
& \operatorname{supp}\left(u^{-}\right) \subset \omega_{-}=\{x \in \omega: h(x)=-1\} .
\end{aligned}
$$

Copyright $@$ ㅇ by SIAM. Unauthorized reproduction of this article is prohibited. 
Hence,

$$
\begin{aligned}
j^{\prime}(u ; v) & =\lim _{\rho \searrow 0} \frac{\|u+\rho v\|_{\mathcal{M}(\omega)}-\|u\|_{\mathcal{M}(\omega)}}{\rho} \\
& =\lim _{\rho \searrow 0} \frac{\left\|u+\rho v_{a}\right\|_{\mathcal{M}(\omega)}+\left\|\rho v_{s}\right\|_{\mathcal{M}(\omega)}-\|u\|_{\mathcal{M}(\omega)}}{\rho} \\
& =\lim _{\rho \searrow 0} \frac{1}{\rho}\left(\int_{\omega}\left|h+\rho g_{v}\right| d|u|-\int_{\omega}|h| d|u|\right)+\left\|v_{s}\right\|_{\mathcal{M}(\omega)} \\
& =\lim _{\rho \searrow 0} \int_{\omega_{+}} \frac{\left|1+\rho g_{v}\right|-1}{\rho} d u^{+}+\lim _{\rho \searrow 0} \int_{\omega_{-}} \frac{\left|-1+\rho g_{v}\right|-1}{\rho} d u^{-}+\left\|v_{s}\right\|_{\mathcal{M}(\omega)} \\
& =\int_{\omega} g_{v} d u+\left\|v_{s}\right\|_{\mathcal{M}(\omega)} .
\end{aligned}
$$

Since the quotients are dominated by $\left|g_{v}\right|$, we could use Lebesgue's dominated convergence theorem in the last identity.

Using the previous propositions we derive the first order optimality conditions for problem $(\mathrm{P})$.

TheOrem 3.4. Suppose that (A1) and (A2) hold and let $\bar{u}$ be a local solution to $(\mathrm{P})$. Then there exists $\bar{\varphi} \in W_{0}^{1, p^{\prime}}(\Omega)$ for some $p^{\prime}>n$ such that

$$
\begin{gathered}
\left\{\begin{array}{cc}
-\Delta \bar{\varphi}+\frac{\partial a}{\partial y}(x, \bar{y}) \bar{\varphi}=\bar{y}-y_{d} & \text { in } \Omega, \\
\bar{\varphi}=0 & \text { on } \Gamma,
\end{array}\right. \\
\alpha\|\bar{u}\|_{\mathcal{M}(\omega)}+\int_{\omega} \bar{\varphi} d \bar{u}=0, \\
\|\bar{\varphi}\|_{C_{0}(\omega)} \begin{cases}=\alpha & \text { if } \bar{u} \neq 0, \\
\leq \alpha & \text { if } \bar{u}=0 .\end{cases}
\end{gathered}
$$

Moreover, if $\bar{u} \neq 0$, then

$$
\begin{aligned}
& \operatorname{supp}\left(\bar{u}^{+}\right) \subset\{x \in \omega: \bar{\varphi}(x)=-\alpha\}, \\
& \operatorname{supp}\left(\bar{u}^{-}\right) \subset\{x \in \omega: \bar{\varphi}(x)=+\alpha\} .
\end{aligned}
$$

Proof. Using Proposition 3.1 and the convexity of $j$ we obtain for every $u \in \mathcal{M}(\omega)$

$$
\begin{aligned}
0 & \leq \lim _{\rho \searrow 0} \frac{J(\bar{u}+\rho(u-\bar{u}))-J(\bar{u})}{\rho} \leq F^{\prime}(\bar{u})(u-\bar{u})+\alpha(j(u)-j(\bar{u})) \\
& =\int_{\omega} \bar{\varphi} d(u-\bar{u})+\alpha(j(u)-j(\bar{u})) .
\end{aligned}
$$

Hence,

$$
\int_{\omega} \frac{-1}{\alpha} \bar{\varphi} d(u-\bar{u})+j(\bar{u}) \leq j(u) \quad \forall u \in \mathcal{M}(\omega)
$$

which implies that $\frac{-1}{\alpha} \bar{\varphi} \in \partial j(\bar{u})$. Now, it is enough to apply Proposition 3.2 to deduce $(3.6)-(3.8)$. 
To prepare for the second order necessary conditions we introduce the critical cone as follows:

$$
C_{\bar{u}}=\left\{v \in \mathcal{M}(\omega): F^{\prime}(\bar{u}) v+\alpha j^{\prime}(\bar{u} ; v)=0\right\} .
$$

It seems natural that the second order optimality conditions must be imposed only on those directions where the directional derivatives vanish. Let us point out some properties of this critical cone.

Proposition 3.5. $C_{\bar{u}}$ is a closed convex cone that can equivalently be expressed in the form

$$
C_{\bar{u}}=\left\{v \in \mathcal{M}(\omega): \int_{\omega} \bar{\varphi} d v_{s}+\alpha\left\|v_{s}\right\|_{\mathcal{M}(\omega)}=0\right\} .
$$

Proof. The cone property and closedness of $C_{\bar{u}}$ are a straightforward consequence of the continuity and positive homogeneity of the mapping $v \rightarrow F^{\prime}(\bar{u}) v+\alpha j^{\prime}(\bar{u} ; v)$. To prove the convexity we first observe that $F^{\prime}(\bar{u}) v+\alpha j^{\prime}(\bar{u} ; v) \geq 0$ for all $v \in \mathcal{M}(\omega)$ since $\bar{u}$ is a local minimum of $J$. Therefore $v$ belongs to $C_{\bar{u}}$ if and only if $F^{\prime}(\bar{u}) v+$ $\alpha j^{\prime}(\bar{u} ; v) \leq 0$. It is therefore enough to use the convexity of the function $v \rightarrow F^{\prime}(\bar{u}) v+$ $\alpha j^{\prime}(\bar{u} ; v)$ to conclude that $C_{\bar{u}}$ is convex. Though the convexity, continuity, and positive homogeneity of $v \rightarrow j^{\prime}(\bar{u} ; v)$ can be easily checked by using the representation given in (3.4), they are also true for any convex and Lipschitz continuous functional $j$; see [4, section 2.4] or [13, Chapter 2].

To prove (3.10), we compute with the aid of (3.1) and (3.4)

$$
\begin{aligned}
F^{\prime}(\bar{u}) v+\alpha j^{\prime}(\bar{u} ; v) & =\int_{\omega} \bar{\varphi} d v+\alpha \int_{\omega} g_{v} d \bar{u}+\alpha\left\|v_{s}\right\|_{\mathcal{M}(\omega)} \\
& =\int_{\omega} \bar{\varphi} g_{v} d|\bar{u}|+\alpha \int_{\omega} g_{v} d \bar{u}+\int_{\omega} \bar{\varphi} d v_{s}+\alpha\left\|v_{s}\right\|_{\mathcal{M}(\omega)} \\
& =\int_{\omega} \bar{\varphi} d v_{s}+\alpha\left\|v_{s}\right\|_{\mathcal{M}(\omega) .}
\end{aligned}
$$

The last identity is a consequence of the fact that $\bar{\varphi} d|\bar{u}|=-\alpha d \bar{u}$, which follows by (3.8).

Remark 3.6. If $\bar{u} \neq 0$, then $\|\bar{\varphi}\|_{\infty}=\alpha$. Consequently, the identity $\int_{\omega} \bar{\varphi} d v_{s}+$ $\alpha\left\|v_{s}\right\|_{\mathcal{M}(\omega)}=0$ implies

$$
\begin{aligned}
& \operatorname{supp}\left(v_{s}^{+}\right) \subset \Omega_{-\alpha}=\{x \in \omega: \bar{\varphi}(x)=-\alpha\} \\
& \operatorname{supp}\left(v_{s}^{-}\right) \subset \Omega_{+\alpha}=\{x \in \omega: \bar{\varphi}(x)=+\alpha\}
\end{aligned}
$$

see [8, Lemma 3.4]. Therefore, the cone $C_{\bar{u}}$ can be expressed in the following way:

$$
C_{\bar{u}}=\left\{v \in \mathcal{M}(\omega): \operatorname{supp} v_{s}^{+} \subset \Omega_{-\alpha} \text { and } \operatorname{supp} v_{s}^{-} \subset \Omega_{+\alpha}\right\} .
$$

Since the support of the absolutely continuous part of $v$ with respect to $|\bar{u}|$ is obviously contained in $\operatorname{supp} \bar{u}$, we deduce with (3.8) that every measure $v \in C_{\bar{u}}$ is supported on the set $\{x \in \Omega:|\bar{\varphi}(x)|=\alpha\}$.

TheOrem 3.7. Suppose that (A1)-(A3). If $\bar{u}$ is a local minimum of $(\mathrm{P})$, then $F^{\prime \prime}(\bar{u}) v^{2} \geq 0$ for all $v \in C_{\bar{u}}$.

Proof. Let $v$ be an element in $C_{\bar{u}}$ and consider the Lebesgue decomposition $d v=g_{v} d|\bar{u}|+d v_{s}$. For every integer $k \geq 1$ we set

$$
g_{v_{k}}(x)=\operatorname{proj}_{[-k,+k]}\left(g_{v}(x)\right) \quad \text { and } \quad d v_{k}=g_{v_{k}} d|\bar{u}|+d v_{s} .
$$

Copyright (c) by SIAM. Unauthorized reproduction of this article is prohibited. 
Then, we have $\left\|v-v_{k}\right\|_{\mathcal{M}(\omega)}=\left\|g_{v}-g_{v_{k}}\right\|_{L^{1}(|\bar{u}|)} \rightarrow 0$ by Lebesgue's dominated convergence theorem. Moreover, since the singular parts of $v_{k}$ and $v$ coincide and $v \in C_{\bar{u}}$, then (3.10) implies that $v_{k} \in C_{\bar{u}}$ for every $k$.

For any $0<\rho<\frac{1}{k}$, following the proof of Proposition 3.3, we find

$$
\begin{aligned}
\frac{j\left(\bar{u}+\rho v_{k}\right)-j(\bar{u})}{\rho} & =\int_{\omega_{+}} \frac{\left|1+\rho g_{v_{k}}\right|-1}{\rho} d u^{+}+\int_{\omega_{-}} \frac{\left|-1+\rho g_{v_{k}}\right|-1}{\rho} d u^{-}+\left\|v_{s}\right\|_{\mathcal{M}(\omega)} \\
& =\int_{\omega} g_{v_{k}} d u+\left\|v_{s}\right\|_{\mathcal{M}(\omega)}=j^{\prime}\left(\bar{u} ; v_{k}\right) .
\end{aligned}
$$

Now, using that $\bar{u}$ is a local minimum of $J$ and making a Taylor expansion we get for every $k$ and $0<\rho<\frac{1}{k}$ the existence of $\theta=\theta(k, \rho)$, with $0<\theta<1$, such that

$0 \leq \frac{J\left(\bar{u}+\rho v_{k}\right)-J(\bar{u})}{\rho}=F^{\prime}(\bar{u}) v_{k}+\frac{\rho}{2} F^{\prime \prime}\left(\bar{u}+\theta \rho v_{k}\right) v_{k}^{2}+\alpha j^{\prime}\left(\bar{u} ; v_{k}\right)=\frac{\rho}{2} F^{\prime \prime}\left(\bar{u}+\theta \rho v_{k}\right) v_{k}^{2}$,

since $v_{k} \in C_{\bar{u}}$. Finally, dividing the last term by $\rho / 2$ and taking the limit when $k \rightarrow \infty$, we get that $F^{\prime \prime}(\bar{u}) v^{2} \geq 0$.

Remark 3.8. Before finishing this section let us observe that we have not made precise the meaning for the local optimality of $\bar{u}$ in Theorems 3.4 and 3.7. In fact, any norm on $\mathcal{M}(\omega)$ leads to the same result, which is not the case for second order sufficient conditions, which will be considered next.

4. Second order sufficient conditions and stability. In this section, $\bar{u}$ will denote an element of $\mathcal{M}(\omega)$, with associated state $\bar{y}$ and adjoint state $\bar{\varphi}$, such that the first order optimality conditions (3.5)-(3.7) hold. Our first goal is to give a second order sufficient condition for the local optimality of $\bar{u}$. To this end we strengthen assumption (A3).

$\left(\mathrm{A} 3^{\prime}\right)$ There exist a constant $C_{a}$ and a function $\phi_{2} \in L^{q_{2}}(\Omega)$ with $q_{2}>2$ such that for a.a. $x \in \Omega$ and for all $s \in \mathbb{R}$

$$
\left\{\begin{array}{l}
\left|\partial_{y}^{2} a(x, s)\right| \leq \phi_{2}(x)+C_{a}|s|^{r} \text { with } 0 \leq r<+\infty \text { if } n=2, \\
\left|\partial_{y}^{2} a(x, s)\right| \leq C_{a} \text { if } n=3 .
\end{array}\right.
$$

Associated to $q_{2}$ and $r$ we introduce $\bar{p}$ as follows. In dimension $n=3$, we take $6 / 5 \leq \bar{p}<3 / 2$ with $\bar{p}$ sufficiently close to $3 / 2$ so that $-\Delta: W_{0}^{1, \bar{p}}(\Omega) \rightarrow W^{-1, \bar{p}}(\Omega)$ is an isomorphism; see [17]. For $n=2$ we take $1 \leq \bar{p}<2$ so that $W_{0}^{1, \bar{p}}(\Omega) \subset L^{q}(\Omega)$, where

$$
q \geq \max \left\{\frac{2 q_{2}}{q_{2}-2}, \frac{4 \bar{r}}{2 \bar{r}-1}\right\} \quad \text { with } \bar{r}=\max \{1, r\}
$$

The reason for this choice of $\bar{p}$ will be clear from the estimates below.

As usual, we have to consider an extended cone of critical directions to formulate a sufficient second order condition for optimality. For every $\tau>0$, we denote

$$
C_{\bar{u}}^{\tau}=\left\{v \in \mathcal{M}(\omega): F^{\prime}(\bar{u}) v+\alpha j^{\prime}(\bar{u} ; v) \leq \tau\left\|z_{v}\right\|_{L^{2}(\Omega)}\right\},
$$

where $z_{v}=G^{\prime}(\bar{u}) v$, with $G$ defined in Theorem 2.2. The second order condition involves this cone as follows: 
(SOSC) There exist positive constants $\kappa, \rho$, and $\tau$ such that

$$
F^{\prime \prime}(u) v^{2} \geq \kappa\left\|z_{v}\right\|_{L^{2}(\Omega)}^{2} \quad \forall v \in C_{\bar{u}}^{\tau} \quad \forall u \in \mathcal{M}(\Omega) \text { with }\|u-\bar{u}\|_{W^{-1, \bar{p}}(\Omega)}<\rho .
$$

Remark 4.1. We point out that the neighborhood for admissible controls in (SOSC) is chosen in $W^{-1, \bar{p}}(\Omega)$ rather than $\mathcal{M}(\omega)$. This is the proper choice, as we show by the following example. Let $x^{0} \in \omega$ and $\left\{x^{k}\right\}_{k=1}^{\infty} \subset \omega$ be such that $x^{k} \rightarrow x^{0}$. Associated to these points we consider the controls $\bar{u}=\delta_{x^{0}}$ and $u_{k}=\delta_{x^{k}}$. It is obvious that $u_{k} \stackrel{*}{\rightarrow} \bar{u}$ in $\mathcal{M}(\omega)$, hence strongly in $W^{-1, \bar{p}}(\Omega)$ and $y_{u_{k}} \rightarrow y_{\bar{u}}$ strongly in $W_{0}^{1, \bar{p}}(\Omega)$. Since $\left\|\bar{u}_{k}\right\|_{\mathcal{M}(\omega)}=\|\bar{u}\|_{\mathcal{M}(\omega)}=1$, we have that $J\left(u_{k}\right) \rightarrow J(\bar{u})$. Let us assume that $\bar{u}$ satisfies the condition (4.2). As we will prove below, $\bar{u}$ is a strict local minimizer of $(\mathrm{P})$ in the sense of the $W^{-1, \bar{p}}(\Omega)$-topology: there exists $\varepsilon \in(0, \rho]$ such that $J(\bar{u})<J(u)$ for $\|u-\bar{u}\|_{W^{-1, \bar{p}}(\Omega)}<\varepsilon, u \neq \bar{u}$. Hence, $J(\bar{u})<J\left(u_{k}\right)$ for all $k$ sufficiently large. If we replace in (4.2) the $W^{-1, \bar{p}}(\Omega)$-ball by the ball $\|u-\bar{u}\|_{\mathcal{M}(\omega)}<\rho$, then we can also prove that $\bar{u}$ is a strict local minimum of $(\mathrm{P})$ in the sense of the $\mathcal{M}(\omega)$-topology. However, if $\varepsilon<2$, this does not allow to guarantee that $J(\bar{u})<J\left(u_{k}\right)$ for $k$ sufficiently large because $\left\|u_{k}-\bar{u}\right\|_{\mathcal{M}(\omega)}=2>\varepsilon$ for every $k$. This example illustrates the fact that the strong topology of $\mathcal{M}(\omega)$ is not the appropriate one for the analysis of the state equation, but rather weaker topologies should be used.

The following theorem implies that (SOSC) is sufficient for strict local optimality of $\bar{u}$.

THEOREM 4.2. Under the assumptions (A1), (A2), (A3'), and (SOSC), there exist positive constants $\varepsilon \leq \rho$ and $\sigma$ such that

$$
J(\bar{u})+\frac{\sigma}{2}\left\|z_{u-\bar{u}}\right\|_{L^{2}(\Omega)}^{2} \leq J(u) \quad \forall\|u-\bar{u}\|_{W^{-1, \bar{p}}(\Omega)}<\varepsilon .
$$

Proof. Let us argue by contradiction and assume that (4.3) does not hold for any $\varepsilon$ and $\sigma$. Then there exists a sequence $\left\{u_{k}\right\}_{k=1}^{\infty} \subset \mathcal{M}(\omega)$ such that

$$
\left\|u_{k}-\bar{u}\right\|_{W^{-1, \bar{p}}(\Omega)}<\frac{1}{k} \text { and } J\left(u_{k}\right)<J(\bar{u})+\frac{1}{2 k}\left\|z_{u_{k}-\bar{u}}\right\|_{L^{2}(\Omega)}^{2} .
$$

Let us prove that $u_{k}-\bar{u} \in C_{\bar{u}}^{\tau}$ for all $k$ sufficiently large. Using the convexity of $j$ we know that

$$
j(u)-j(\bar{u}) \geq j^{\prime}(\bar{u} ; u-\bar{u}) \quad \forall u \in \mathcal{M}(\omega) .
$$

Combining (4.4) and (4.5), a Taylor expansion of $F$ around $\bar{u}$ leads to

$$
\frac{1}{2 k}\left\|z_{u_{k}-\bar{u}}\right\|_{L^{2}(\Omega)}^{2}>F^{\prime}(\bar{u})\left(u_{k}-\bar{u}\right)+\alpha j^{\prime}\left(\bar{u} ; u_{k}-\bar{u}\right)+\frac{1}{2} F^{\prime \prime}\left(u_{\theta}\right)\left(u_{k}-\bar{u}\right)^{2},
$$

where $u_{\theta}=\bar{u}+\theta\left(u_{k}-\bar{u}\right), 0<\theta<1$. Using (3.3), (2.9), and (2.10) we get

$$
F^{\prime \prime}\left(u_{\theta}\right)\left(u_{k}-\bar{u}\right)^{2}=\int_{\Omega}\left[\left(y_{\theta}-y_{d}\right) w_{\theta}+z_{\theta, u_{k}-\bar{u}}^{2}\right] d x,
$$

where $y_{\theta}=G\left(u_{\theta}\right), z_{\theta, u_{k}-\bar{u}}=G^{\prime}\left(u_{\theta}\right)\left(u_{k}-\bar{u}\right)$, and $w_{\theta}=G^{\prime \prime}\left(u_{\theta}\right)\left(u_{k}-\bar{u}\right)^{2}$. From Lemma 4.3 below, we have for every $k$

$$
\left\|z_{\theta, u_{k}-\bar{u}}\right\|_{L^{2}(\Omega)} \leq C\left\|z_{u_{k}-\bar{u}}\right\|_{L^{2}(\Omega)} \text { and }\left\|w_{\theta}\right\|_{L^{2}(\Omega)} \leq C\left\|z_{u_{k}-\bar{u}}\right\|_{W_{0}^{1, \bar{p}}(\Omega)}\left\|z_{u_{k}-\bar{u}}\right\|_{L^{2}(\Omega)},
$$

where $z_{u_{k}-\bar{u}}=G^{\prime}(\bar{u})\left(u_{k}-\bar{u}\right)$. Because of our choice of $\bar{p}$ and (4.4), we have

$$
\left\|z_{u_{k}-\bar{u}}\right\|_{L^{2}(\Omega)} \leq C\left\|z_{u_{k}-\bar{u}}\right\|_{W_{0}^{1, \bar{p}}(\Omega)} \leq C\left\|u_{k}-\bar{u}\right\|_{W^{-1, \bar{p}}(\Omega)}<\frac{C}{k} .
$$

Copyright $@$ by SIAM. Unauthorized reproduction of this article is prohibited. 
The last inequalities imply

$$
\begin{aligned}
\left|F^{\prime \prime}\left(u_{\theta}\right)\left(u_{k}-\bar{u}\right)^{2}\right| & \leq\left\|y_{\theta}-y_{d}\right\|_{L^{2}(\Omega)}\left\|w_{\theta}\right\|_{L^{2}(\Omega)}+\left\|z_{\theta, u_{k}-\bar{u}}\right\|_{L^{2}(\Omega)}^{2} \\
& \leq C\left(\left\|y_{\theta}-y_{d}\right\|_{L^{2}(\Omega)}\left\|z_{u_{k}-\bar{u}}\right\|_{W_{0}^{1, \bar{p}}(\Omega)}+\left\|z_{u_{k}-\bar{u}}\right\|_{L^{2}(\Omega)}\right)\left\|z_{u_{k}-\bar{u}}\right\|_{L^{2}(\Omega)} \\
& \leq \frac{C}{k}\left\|z_{u_{k}-\bar{u}}\right\|_{L^{2}(\Omega)} .
\end{aligned}
$$

Combining this inequality and (4.6) we deduce

$$
F^{\prime}(\bar{u})\left(u_{k}-\bar{u}\right)+\alpha j^{\prime}\left(\bar{u} ; u_{k}-\bar{u}\right) \leq \frac{C}{k}\left\|z_{u_{k}-\bar{u}}\right\|_{L^{2}(\Omega)} \quad \forall k .
$$

Hence, for $C / k<\tau$, we get that $u_{k}-\bar{u} \in C_{\bar{u}}^{\tau}$. Moreover, from (3.7) and (3.11) it follows that

$$
F^{\prime}(\bar{u})\left(u_{k}-\bar{u}\right)+\alpha j^{\prime}\left(\bar{u} ; u_{k}-\bar{u}\right) \geq 0 .
$$

Finally, from this inequality, (4.6) and (4.2), and observing that $\left\|u_{\theta}-\bar{u}\right\|_{W^{-1, \bar{p}}(\Omega)} \leq$ $1 / k<\varepsilon$ for $k$ large, it follows that

$$
\frac{1}{2 k}\left\|z_{u_{k}-\bar{u}}\right\|_{L^{2}(\Omega)}^{2} \geq \frac{1}{2} F^{\prime \prime}\left(u_{\theta}\right)\left(u_{k}-\bar{u}\right)^{2} \geq \frac{\kappa}{2}\left\|z_{u_{k}-\bar{u}}\right\|_{L^{2}(\Omega)}^{2} .
$$

Since (4.4) implies that $u_{k} \neq \bar{u}$, the above inequality gives the contradiction.

LEMma 4.3. Let $u, \bar{u}, v \in \mathcal{M}(\omega)$ and $y, \bar{y}, z, \bar{z}, w \in V(\Omega)$ with $y=G(u), \bar{y}=$ $G(\bar{u}), z=G^{\prime}(u) v, \bar{z}=G^{\prime}(\bar{u}) v$, and $w=G^{\prime \prime}(u) v^{2}$. Then, for every $M>0$ there exist a positive constant $C_{M}$ independent of $v$ such that the inequalities

$$
\|z\|_{L^{2}(\Omega)} \leq C_{M}\|\bar{z}\|_{L^{2}(\Omega)} \quad \text { and } \quad\|w\|_{L^{2}(\Omega)} \leq C_{M}\|\bar{z}\|_{W_{0}^{1, \bar{p}}(\Omega)}\|\bar{z}\|_{L^{2}(\Omega)}
$$

hold for every $\|u\|_{W^{-1, \bar{p}}(\Omega)} \leq M$ and $\|\bar{u}\|_{W^{-1, \bar{p}}(\Omega)} \leq M$.

Proof. According to (2.9) and (2.10), $z, \bar{z}$, and $w$ satisfy the equations

$$
\begin{aligned}
& \left\{\begin{aligned}
-\Delta z+\frac{\partial a}{\partial y}(x, y) z & =v & & \text { in } \Omega, \\
z & =0 & & \text { on } \Gamma,
\end{aligned}\right. \\
& \left\{\begin{aligned}
-\Delta \bar{z}+\frac{\partial a}{\partial y}(x, \bar{y}) \bar{z} & =v & & \text { in } \Omega, \\
\bar{z} & =0 & & \text { on } \Gamma,
\end{aligned}\right.
\end{aligned}
$$

and

$$
\left\{\begin{aligned}
-\Delta w+\frac{\partial a}{\partial y}(x, y) w+\frac{\partial^{2} a}{\partial y^{2}}(x, y) z^{2} & =0 & & \text { in } \Omega, \\
w & =0 & & \text { on } \Gamma .
\end{aligned}\right.
$$

Subtracting (4.9) from (4.8) and applying the mean value theorem, we get for some $\hat{y}=\bar{y}+\theta(y-\bar{y})$ with $0 \leq \theta(x) \leq 1$,

$$
\left\{\begin{aligned}
-\Delta(z-\bar{z})+\frac{\partial a}{\partial y}(x, y)(z-\bar{z})+\frac{\partial^{2} a}{\partial y^{2}}(x, \hat{y})(y-\bar{y}) \bar{z}=0 & \text { in } \Omega, \\
z-\bar{z}=0 & \text { on } \Gamma .
\end{aligned}\right.
$$

Copyright $@$ ㅇ by SIAM. Unauthorized reproduction of this article is prohibited. 
From this equation and recalling our choice of $\bar{p}$ we have

$$
\begin{aligned}
\|z-\bar{z}\|_{L^{2}(\Omega)} & \leq C\|z-\bar{z}\|_{W_{0}^{1, \bar{p}}(\Omega)} \leq C\left\|\frac{\partial^{2} a}{\partial y^{2}}(x, \hat{y})(y-\bar{y}) \bar{z}\right\|_{W^{-1, \bar{p}}(\Omega)} \\
& \leq C\left\|\frac{\partial^{2} a}{\partial y^{2}}(x, \hat{y})(y-\bar{y}) \bar{z}\right\|_{L^{1}(\Omega)} \leq C\left\|\frac{\partial^{2} a}{\partial y^{2}}(x, \hat{y})(y-\bar{y})\right\|_{L^{2}(\Omega)}\|\bar{z}\|_{L^{2}(\Omega)}
\end{aligned}
$$

In dimension 3, Assumption (A3') implies the boundedness of $\partial_{y}^{2} a(x, \hat{y})$ and consequently

$$
\|z-\bar{z}\|_{L^{2}(\Omega)} \leq C\|y-\bar{y}\|_{W_{0}^{1, \bar{p}}(\Omega)}\|\bar{z}\|_{L^{2}(\Omega)},
$$

where we have estimated the $L^{2}(\Omega)$-norm of $y-\bar{y}$ by the $W_{0}^{1, \bar{p}}(\Omega)$-norm. Hence, in dimension 3 , the first inequality in (4.7) follows from the triangle inequality and (4.12).

To obtain the estimate (4.12) for dimension 2, we use again assumption (A3') to get

$$
\left|\frac{\partial^{2} a}{\partial y^{2}}(x, \hat{y})\right| \leq \phi_{2}(x)+C_{a}|\hat{y}|^{r} \leq \phi_{2}(x)+C_{a}(1+|\hat{y}|)^{\bar{r}} .
$$

Then, our choice of $\bar{p}$ and Hölder's inequality imply

$$
\begin{aligned}
& \left\|\frac{\partial^{2} a}{\partial y^{2}}(x, \hat{y})(y-\bar{y})\right\|_{L^{2}(\Omega)} \\
& \quad \leq C\left(\left\|\phi_{2}\right\|_{L^{q_{2}(\Omega)}}+\|1+|\hat{y}|\|_{L^{2 \bar{r}}(\Omega)}\right)\|y-\bar{y}\|_{L^{q}(\Omega)} \leq C\|y-\bar{y}\|_{W_{0}^{1, \bar{p}}(\Omega)} .
\end{aligned}
$$

We proceed as in the three-dimensional case to prove the first estimate in (4.7).

Let us also notice that from (4.11) and (4.12) we infer for $n=2$ or 3

$$
\|z-\bar{z}\|_{W_{0}^{1, \bar{p}}(\Omega)} \leq C\|\bar{z}\|_{L^{2}(\Omega)} \leq C\|\bar{z}\|_{W_{0}^{1, \bar{p}}(\Omega)} .
$$

Once again, the triangle inequality leads to

$$
\|z\|_{W_{0}^{1, \bar{p}}(\Omega)} \leq C\|\bar{z}\|_{W_{0}^{1, \bar{p}}(\Omega)} .
$$

Finally, to prove the second inequality of (4.7) we use (4.10) and the first inequality of (4.7) to obtain

$$
\begin{aligned}
\|w\|_{L^{2}(\Omega)} & \leq C\|w\|_{W_{0}^{1, \bar{p}}(\Omega)} \leq C\left\|\frac{\partial^{2} a}{\partial y^{2}}(x, \hat{y}) z^{2}\right\|_{L^{1}(\Omega)} \\
& \leq C\left\|\frac{\partial^{2} a}{\partial y^{2}}(x, \hat{y}) z\right\|_{L^{2}(\Omega)}\|z\|_{L^{2}(\Omega)} \leq C\left\|\frac{\partial^{2} a}{\partial y^{2}}(x, \hat{y}) z\right\|_{L^{2}(\Omega)}\|\bar{z}\|_{L^{2}(\Omega)} .
\end{aligned}
$$

Replacing $y-\bar{y}$ by $z$, we can argue as above to estimate $\partial_{y}^{2} a(x, \hat{y}) z$ in $L^{2}(\Omega)$. Therefore, with (4.13) we conclude

$$
\|w\|_{L^{2}(\Omega)} \leq C\|z\|_{W_{0}^{1, \bar{p}}(\Omega)}\|\bar{z}\|_{L^{2}(\Omega)} \leq C\|\bar{z}\|_{W_{0}^{1, \bar{p}}(\Omega)}\|\bar{z}\|_{L^{2}(\Omega)} .
$$


Remark 4.4. The reader may observe that the second order sufficient optimality condition (4.2) is not imposed at the point $\bar{u}$ as usual. It is imposed for every $u$ in a certain ball around $\bar{u}$. The reason for this stronger assumption is that we have not been able to prove that given any $\varepsilon>0$ there exists $\rho>0$ such that

$$
\left|\left[F^{\prime \prime}(u)-F^{\prime \prime}(\bar{u})\right] v^{2}\right| \leq \varepsilon\left\|z_{v}\right\|_{L^{2}(\Omega)} \quad \forall\|u-\bar{u}\|_{W^{-1, \bar{p}}(\Omega)}<\rho \text { and } \forall v \in C_{\bar{u}}^{\tau} .
$$

COROLlary 4.5. Under the assumptions of Theorem 4.2, there exists a constant $\hat{\sigma}>0$ independent of $u$ such that

$$
J(\bar{u})+\frac{\hat{\sigma}}{2}\|y(u)-\bar{y}\|_{L^{2}(\Omega)}^{2} \leq J(u) \quad \forall\|u-\bar{u}\|_{W^{-1, \bar{p}}(\Omega)}<\varepsilon,
$$

where $y(u)=G(u)$.

Proof. For simplification, we write $y=y(u)$. Subtracting the equations satisfied by $y$ and $\bar{y}$ we get for $\hat{y}=\bar{y}+\theta(y-\bar{y}), \theta$ being a Lebesgue measurable function such that $0 \leq \theta(x) \leq 1$,

$$
\left\{\begin{aligned}
-\Delta(y-\bar{y})+\frac{\partial a}{\partial y}(x, \hat{y})(y-\bar{y}) & =u-\bar{u} & & \text { in } \Omega, \\
y-\bar{y} & =0 & & \text { on } \Gamma .
\end{aligned}\right.
$$

Using the identity

$$
\frac{\partial a}{\partial y}(x, \bar{y})=\frac{\partial a}{\partial y}(x, \hat{y})-\theta(x) \frac{\partial^{2} a}{\partial y^{2}}(x, \tilde{y})(y-\bar{y})
$$

in the equation of $z_{u-\bar{u}}$ we get

$$
\left\{\begin{aligned}
-\Delta z_{u-\bar{u}}+\frac{\partial a}{\partial y}(x, \hat{y}) z_{u-\bar{u}}-\theta(x) \frac{\partial^{2} a}{\partial y^{2}}(x, \tilde{y})(y-\bar{y}) z_{u-\bar{u}} & =u-\bar{u} & & \text { in } \Omega, \\
z_{u-\bar{u}} & =0 & & \text { on } \Gamma .
\end{aligned}\right.
$$

Subtracting the equations for $y-\bar{y}$ and $z_{u-\bar{u}}$, and setting $\xi=y-\bar{y}-z_{u-\bar{u}}$, we obtain

$$
\left\{\begin{aligned}
-\Delta \xi+\frac{\partial a}{\partial y}(x, \hat{y}) \xi+\theta(x) \frac{\partial^{2} a}{\partial y^{2}}(x, \tilde{y})(y-\bar{y}) z_{u-\bar{u}}=0 & & \text { in } \Omega, \\
\xi=0 & & \text { on } \Gamma .
\end{aligned}\right.
$$

Proceeding as in the proof of Lemma 4.3 we find

$$
\begin{aligned}
\|\xi\|_{L^{2}(\Omega)} & \leq C\|y-\bar{y}\|_{W_{0}^{1, \bar{p}}(\Omega)}\left\|z_{u-\bar{u}}\right\|_{L^{2}(\Omega)} \\
& \leq C\|u-\bar{u}\|_{W^{-1, \bar{p}}(\Omega)}\left\|z_{u-\bar{u}}\right\|_{L^{2}(\Omega)} \leq C \varepsilon\left\|z_{u-\bar{u}}\right\|_{L^{2}(\Omega)} .
\end{aligned}
$$

Finally, from the triangle inequality we have

$$
\|y-\bar{y}\|_{L^{2}(\Omega)} \leq\|\xi\|_{L^{2}(\Omega)}+\left\|z_{u-\bar{u}}\right\|_{L^{2}(\Omega)} \leq(C \varepsilon+1)\left\|z_{u-\bar{u}}\right\|_{L^{2}(\Omega)},
$$

which with (4.3) leads to (4.14) for $\hat{\sigma}=\sigma /(C \varepsilon+1)^{2}$.

The rest of the section is dedicated to the stability analysis of the control problem (P) with respect to perturbations of the desired state $y_{d}$. More precisely, for $\delta>0$ consider the problems

$$
\left(\mathrm{P}_{\delta}\right) \quad \min _{u \in \mathcal{M}(\omega)} J_{\delta}(u)=\frac{1}{2}\left\|y-y_{d, \delta}\right\|_{L^{2}(\Omega)}^{2}+\alpha\|u\|_{\mathcal{M}(\omega)},
$$


where $y$ is the solution to $(1.2)$ and $\left\{y_{d, \delta}\right\}_{\delta>0} \subset L^{2}(\Omega)$ satisfies

$$
\left\|y_{d}-y_{d, \delta}\right\|_{L^{2}(\Omega)} \leq \delta .
$$

We denote by $u_{\delta}$ local solutions to $\left(\mathrm{P}_{\delta}\right)$ with associated states $y_{\delta}$. We have the following approximation theorem as $\delta \rightarrow 0$.

THEOREM 4.6. Suppose that (A1) holds. Then every family $\left\{u_{\delta}\right\}_{\delta>0}$ of global solutions is bounded in $\mathcal{M}(\omega)$ and every weak ${ }^{*}$ subsequential limit $\bar{u}$ is a global solution to $(\mathrm{P})$. The convergence properties

$$
\left\|u_{\delta}\right\|_{\mathcal{M}(\omega)} \rightarrow\|\bar{u}\|_{\mathcal{M}(\omega)}, \quad\left\|u_{\delta}-\bar{u}\right\|_{W^{-1, p}(\Omega)} \rightarrow 0 \quad \text { and }\left\|y_{\delta}-\bar{y}\right\|_{W_{0}^{1, p}(\Omega)} \rightarrow 0
$$

hold for every $p<n /(n-1)$. Conversely, for every strict local minimum $\bar{u}$ of $(\mathrm{P})$ in the $W^{-1, p}(\Omega)$ (or $\mathcal{M}(\omega)$ ) sense there exists a sequence of local solutions $\left\{u_{\delta}\right\}_{\delta>0}$ of $\left(\mathrm{P}_{\delta}\right)$ such that (4.16) holds.

Proof. Denote by $y_{0}$ the solution to (1.2) associated to the control $u=0$. Then, using (4.15) we get

$$
\alpha\left\|u_{\delta}\right\|_{\mathcal{M}(\omega)} \leq J_{\delta}\left(u_{\delta}\right) \leq J_{\delta}(0) \leq \frac{1}{2}\left(\left\|y_{0}-y_{d}\right\|_{L^{2}(\Omega)}+\delta\right)^{2},
$$

which proves the boundedness of $\left\{u_{\delta}\right\}_{\delta>0}$. Hence, taking a subsequence, if necessary, we have $u_{\delta} \stackrel{*}{\rightarrow} \bar{u}$ in $\mathcal{M}(\omega)$. From the compactness of the embedding $\mathcal{M}(\omega) \subset W^{-1, p}(\Omega)$ for every $p<n /(n-1)$, we get the strong convergence in $W^{-1, p}(\Omega)$ and the strong convergence $y_{\delta} \rightarrow \bar{y}$ in $W_{0}^{1, p}(\Omega)$, where $\bar{y}=G(\bar{u})$. Let us prove that $\bar{u}$ is a global solution to $(\mathrm{P})$. From the stated convergence properties and (4.15) we get for every $u \in \mathcal{M}(\omega)$

$$
J(\bar{u}) \leq \liminf _{\delta \rightarrow 0} J_{\delta}\left(u_{\delta}\right) \leq \limsup _{\delta \rightarrow 0} J_{\delta}\left(u_{\delta}\right) \leq \limsup _{\delta \rightarrow 0} J_{\delta}(u)=J(u),
$$

which proves the optimality of $\bar{u}$. In particular, taking $u=\bar{u}$ we deduce from the above inequalities that $J_{\delta}\left(u_{\delta}\right) \rightarrow J(\bar{u})$, which implies that $\left\|u_{\delta}\right\|_{\mathcal{M}(\omega)} \rightarrow\|\bar{u}\|_{\mathcal{M}(\omega)}$.

Conversely, let $\bar{u}$ be a strict local solution of (P). Then, for some $\varepsilon>0, \bar{u}$ is the unique global solution of the problem

$$
\left(\mathrm{P}_{\varepsilon}\right) \quad \min _{u \in U_{\varepsilon}} J(u)=\frac{1}{2}\left\|y-y_{d}\right\|_{L^{2}(\Omega)}^{2}+\alpha\|u\|_{\mathcal{M}(\omega)}
$$

with $U_{\varepsilon}=\left\{u \in \mathcal{M}(\omega):\|u-\bar{u}\|_{W^{-1, p}(\Omega)} \leq \varepsilon\right\}$. We also introduce the perturbed problems

$$
\left(\mathrm{P}_{\varepsilon, \delta}\right) \quad \min _{u \in U_{\varepsilon}} J_{\delta}(u)=\frac{1}{2}\left\|y-y_{d, \delta}\right\|_{L^{2}(\Omega)}^{2}+\alpha\|u\|_{\mathcal{M}(\omega)} .
$$

Observe that the compactness of the embedding $\mathcal{M}(\omega) \in W^{-1, p}(\Omega)$ implies that $U_{\varepsilon}$ is sequentially weakly* closed in $\mathcal{M}(\omega)$. This implies the existence of global solutions $u_{\delta}$ to problems $\left(\mathrm{P}_{\varepsilon, \delta}\right)$. Now, we can argue as in the first part of the theorem to deduce (4.16). In this case we replace the inequality $J_{\delta}\left(u_{\delta}\right) \leq J_{\delta}(0)$ by $J_{\delta}\left(u_{\delta}\right) \leq J_{\delta}(\bar{u})$. As a consequence we have that $\left\|u_{\delta}-\bar{u}\right\|_{W^{-1, p}(\Omega)}<\varepsilon$ for $\delta$ sufficiently small, which shows that $u_{\delta}$ is a local solution of $\left(\mathrm{P}_{\delta}\right)$.

To get a rate of convergence for the states $\left\{y_{\delta}\right\}_{\delta>0}$ to $\bar{y}$ we use (SOSC). Let us fix a local solution $\bar{u}$ of (P) satisfying (SOSC) and let $\varepsilon>0$ be given by Theorem 4.2. We 
know from the proof of Theorem 4.6 that there exists a sequence $\left\{u_{\delta}\right\}_{\delta>0}$ converging to $\bar{u}$ in the sense of (4.16) and such that every $u_{\delta}$ is a minimum of $J_{\delta}$ in the ball $\|u-\bar{u}\|_{W^{-1, \bar{p}}(\Omega)}<\varepsilon$.

THEOREM 4.7. With the above notation and assuming (A1), (A2), and (A3'), there exists a constant $C$ independent of $\delta$ such that

$$
\left\|y_{\delta}-\bar{y}\right\|_{L^{2}(\Omega)} \leq C \sqrt{\delta} \quad \text { and } \quad\left|\left\|u_{\delta}\right\|_{\mathcal{M}(\omega)}-\|\bar{u}\|_{\mathcal{M}(\omega)}\right| \leq C \sqrt{\delta} .
$$

Proof. Using (4.14), the optimality of $u_{\delta}$, and (4.15) it follows that

$$
\begin{aligned}
\frac{\hat{\sigma}}{2}\left\|y_{\delta}-\bar{y}\right\|_{L^{2}(\Omega)}^{2} \leq & J\left(u_{\delta}\right)-J(\bar{u}) \\
\leq & J_{\delta}\left(u_{\delta}\right)-J(\bar{u})+\frac{1}{2}\left\{\left\|y_{\delta}-y_{d}\right\|_{L^{2}(\Omega)}^{2}-\left\|y_{\delta}-y_{d, \delta}\right\|_{L^{2}(\Omega)}^{2}\right\} \\
\leq & J_{\delta}(\bar{u})-J(\bar{u})+\frac{1}{2}\left\{\left\|y_{\delta}-y_{d}\right\|_{L^{2}(\Omega)}^{2}-\left\|y_{\delta}-y_{d, \delta}\right\|_{L^{2}(\Omega)}^{2}\right\} \\
= & \frac{1}{2}\left\{\left\|\bar{y}-y_{d, \delta}\right\|_{L^{2}(\Omega)}^{2}-\left\|\bar{y}-y_{d}\right\|_{L^{2}(\Omega)}^{2}\right\} \\
& +\frac{1}{2}\left\{\left\|y_{\delta}-y_{d}\right\|_{L^{2}(\Omega)}^{2}-\left\|y_{\delta}-y_{d, \delta}\right\|_{L^{2}(\Omega)}^{2}\right\} \\
\leq & \frac{1}{2}\left\{\left\|2 \bar{y}-\left(y_{d, \delta}+y_{d}\right)\right\|_{L^{2}(\Omega)}+\left\|2 y_{\delta}-\left(y_{d, \delta}+y_{d}\right)\right\|_{L^{2}(\Omega)}\right\} \delta \leq C \delta,
\end{aligned}
$$

which proves the first estimate of the theorem. For the second estimate we use the optimality of $\bar{u}$ and $u_{\delta}$ to get

$$
\begin{aligned}
0 & \leq J\left(u_{\delta}\right)-J(\bar{u})=\frac{1}{2}\left(\left\|y_{\delta}-y_{d}\right\|_{L^{2}(\Omega)}^{2}-\left\|\bar{y}-y_{d}\right\|_{L^{2}(\Omega)}^{2}\right)+\alpha\left(\left\|u_{\delta}\right\|_{\mathcal{M}(\omega)}-\|\bar{u}\|_{\mathcal{M}(\omega)}\right) \\
& \leq C \sqrt{\delta}+\alpha\left(\left\|u_{\delta}\right\|_{\mathcal{M}(\omega)}-\|\bar{u}\|_{\mathcal{M}(\omega)}\right)
\end{aligned}
$$

and

$$
\begin{aligned}
0 & \leq J_{\delta}(\bar{u})-J_{\delta}\left(u_{\delta}\right)=\frac{1}{2}\left(\left\|\bar{y}-y_{d, \delta}\right\|_{L^{2}(\Omega)}^{2}-\left\|y_{\delta}-y_{d, \delta}\right\|_{L^{2}(\Omega)}^{2}\right)+\alpha\left(\|\bar{u}\|_{\mathcal{M}(\omega)}-\left\|u_{\delta}\right\|_{\mathcal{M}(\omega)}\right) \\
& \leq C \sqrt{\delta}+\alpha\left(\|\bar{u}\|_{\mathcal{M}(\omega)}-\left\|u_{\delta}\right\|_{\mathcal{M}(\omega)}\right) .
\end{aligned}
$$

Combining these two estimates the second inequality follows.

Remark 4.8. Consider a perturbation in the state equation of the following type:

$$
\left\{\begin{aligned}
-\Delta y+a(x, y) & =u+f_{\delta} & & \text { in } \Omega, \\
y & =0 & & \text { on } \Gamma,
\end{aligned}\right.
$$

where

$$
\left\|f_{\delta}\right\|_{L^{1}(\Omega)} \leq \delta .
$$

Associated to these perturbed state equations we can define control problems $\left(\mathrm{P}_{\delta}\right)$, analogous to problem $(\mathrm{P})$ with solutions $u_{\delta}$. The previous analysis can be repeated to get the estimates of Theorem 4.7. In this argumentation it is enough to establish that for every control $u \in \mathcal{M}(\omega)$ with corresponding states $y_{\delta}$ and $y$, solutions to (4.17) and (1.2), respectively, satisfy

$$
\left\|y_{\delta}-y\right\|_{W_{0}^{1, p}(\Omega)} \leq C\left\|f_{\delta}\right\|_{L^{1}(\Omega)} \leq C \delta .
$$

Copyright $@$ by SIAM. Unauthorized reproduction of this article is prohibited. 
5. A regularity result. The goal of this section is to prove a regularity result for the optimal controls and the associated states assuming that $y_{d} \in L^{\infty}(\Omega)$ and $\omega=\Omega$. A similar result was obtained for linear state equations in [18]. The assumption that the control domain coincides with the observation domain can be restrictive for genuine control problems. However, it is an efficient way to determine the optimal placement of actuators.

THEOREM 5.1. Let $\bar{u}$ satisfy the first order optimality conditions (3.5)-(3.7) with associated state $\bar{y}$. Assume that $\omega=\Omega, y_{d} \in L^{\infty}(\Omega)$, and (A1) and (A2) hold with $\phi_{0} \in L^{q_{1}}(\Omega)$, $q_{1}$, and $r$ given by (2.7). Then, we have that $\bar{y} \in H_{0}^{1}(\Omega) \cap L^{\infty}(\Omega)$, $\bar{u} \in \mathcal{M}(\Omega) \cap H^{-1}(\Omega)$, and

$$
\begin{aligned}
& \|\bar{y}\|_{L^{\infty}(\Omega)} \leq C\left(\left\|y_{d}\right\|_{L^{\infty}(\Omega)}+\|a(\cdot, 0)\|_{L^{q_{1}}(\Omega)}\right) \\
& \|\bar{y}\|_{H_{0}^{1}(\Omega)} \leq C\left(\left\|y_{d}\right\|_{L^{\infty}(\Omega)}\|\bar{u}\|_{\mathcal{M}(\Omega)}+\|a(\cdot, 0)\|_{L^{q_{1}}(\Omega)}^{2}\right)^{1 / 2}
\end{aligned}
$$

for a constant $C$ independent of $\bar{u}$.

The reader can easily check that (5.1) and (5.2) hold if $\bar{u}=0$. Hence, we assume that $\bar{u} \neq 0$. Let us introduce some notation. We decompose $\bar{y}=\bar{w}+\bar{\zeta}^{+}-\bar{\zeta}^{-}$with

$$
\begin{gathered}
\left\{\begin{array} { r l } 
{ - \Delta \overline { \zeta } ^ { + } = \overline { u } ^ { + } } & { \text { in } \Omega , } \\
{ \overline { \zeta } ^ { + } = 0 } & { \text { on } \Gamma , }
\end{array} \quad \left\{\begin{array}{rl}
-\Delta \bar{\zeta}^{-}=\bar{u}^{-} & \text {in } \Omega, \\
\bar{\zeta}^{-}=0 & \text { on } \Gamma,
\end{array}\right.\right. \\
\left\{\begin{aligned}
-\Delta \bar{w}+g(x, \bar{w})=f & \text { in } \Omega, \\
\bar{w}=0 & \text { on } \Gamma
\end{aligned}\right.
\end{gathered}
$$

with

$$
f(x)=-a\left(x, \bar{\zeta}^{+}(x)-\bar{\zeta}^{-}(x)\right) \text { and } g(x, t)=a\left(x, t+\bar{\zeta}^{+}(x)-\bar{\zeta}^{-}(x)\right)+f(x) .
$$

Taking into account that $\bar{\zeta}^{+}-\bar{\zeta}^{-} \in V(\Omega) \subset L^{p}(\Omega)$ for all $p<+\infty$ if $n=2$, and all $p<3$ if $n=3$, that $\phi_{0} \in L^{q_{1}}(\Omega)$, with $q_{1}>n / 2, r<2$ if $n=3$, and assumption (A1), we conclude that the function $f \in L^{q}(\Omega)$ for some $q_{1} \geq q>n / 2$. In dimension $n=3$, we take $q>3 / 2$ and such that $r q<3$. Hence, the solution $\bar{w}$ of (5.4) belongs to $H_{0}^{1}(\Omega) \cap C_{0}(\Omega)$; see [15, Chapter 8].

We also consider the functions

$$
\bar{\zeta}^{+*}(x)=\int_{\Omega} \mathcal{G}(x, \xi) d \bar{u}^{+}(\xi), \quad \bar{\zeta}^{-*}(x)=\int_{\Omega} \mathcal{G}(x, \xi) d \bar{u}^{-}(\xi), \quad \text { and } \quad \bar{\zeta}^{*}=\bar{\zeta}^{+*}-\bar{\zeta}^{-*},
$$

where $\mathcal{G}$ denotes the Green's function for the Dirichlet problem in $\Omega$ associated to the Laplace operator. The reader should notice that $\bar{\zeta}^{+}\left(\bar{\zeta}^{-}\right)$denotes a class of measurable functions, while $\bar{\zeta}^{+*}\left(\bar{\zeta}^{-*}\right)$ is a particular selection in this class well defined at every point of $\Omega$, that could take the value $+\infty$ at some points. Define $\bar{y}^{*}=\bar{w}+\bar{\zeta}^{*}$.

The proof will utilize the following three lemmas.

LEMma 5.2. The following properties hold:

$$
\begin{cases}\bar{y}^{*}(x) \leq+\left\|y_{d}\right\|_{L^{\infty}(\Omega)} & \forall x \in \operatorname{supp} \bar{u}^{+} \\ \bar{y}^{*}(x) \geq-\left\|y_{d}\right\|_{L^{\infty}(\Omega)} & \forall x \in \operatorname{supp} \bar{u}^{-} .\end{cases}
$$

Proof. Let us prove the first inequality, the proof of the second being analogous. Let us assume that the inequality is false. Then, there exists a point $x_{0} \in \operatorname{supp} \bar{u}^{+}$ such that $\bar{y}^{*}\left(x_{0}\right)>\left\|y_{d}\right\|_{L^{\infty}(\Omega)}$. From (3.8) we know that the supports of $\bar{u}^{+}$and $\bar{u}^{-}$ 
are disjoint compact sets. Then we have that $\bar{\zeta}^{-*}$ is continuous in a neighborhood of $\operatorname{supp} \bar{u}^{+}$; see [21]. Moreover, $\bar{w}$ is continuous and $\bar{\zeta}^{+*}$ is lower semicontinuous in $\Omega$. Hence, $\bar{y}^{*}$ is also lower semicontinuous in a neighborhood of supp $\bar{u}^{+}$. Therefore, there exists a ball $B_{\rho}\left(x_{0}\right)$ such that $\bar{y}^{*}(x)>\left\|y_{d}\right\|_{L^{\infty}(\Omega)}$ for every $x \in B_{\rho}\left(x_{0}\right)$. Then, (3.5) leads to

$$
-\Delta \bar{\varphi}+\frac{\partial a}{\partial y}(x, \bar{y}) \bar{\varphi}=\bar{y}-y_{d}>0 \quad \text { a.e. in } B_{\rho}\left(x_{0}\right) .
$$

From (3.8), we know that $\bar{\varphi}\left(x_{0}\right)=-\alpha$; consequently $\bar{\varphi}$ cannot be constant in the ball $B_{\rho}\left(x_{0}\right)$ because the left-hand side would be nonpositive and the right-hand side strictly positive. Therefore, an application of the maximum principle shows that there exists $x^{\prime} \in \partial B_{\rho}\left(x_{0}\right)$ such that $\bar{\varphi}\left(x^{\prime}\right)<\bar{\varphi}\left(x_{0}\right)=-\alpha$, which contradicts (3.7).

Remark 5.3. In the case $\omega \neq \Omega$, (3.7) says that $\|\bar{\varphi}\|_{C_{0}(\omega)} \leq \alpha$, but $|\bar{\varphi}|$ can be bigger than $\alpha$ outside $\omega$. As a consequence, the proof of Lemma 5.2 is not valid. Indeed, $\varphi\left(x_{0}\right)=-\alpha$ and $\bar{\varphi}\left(x^{\prime}\right)<-\alpha$, with $x^{\prime} \in \partial B_{\rho}\left(x_{0}\right)$, is not a contradiction. Since this lemma is crucial in the proof of Theorem 5.1, it is our opinion that the regularity result is not valid for $\omega \neq \Omega$.

Lemma 5.4. We have that $\bar{y} \in H_{0}^{1}(\Omega) \cap L^{\infty}(\Omega)$ and $\bar{u} \in H^{-1}(\Omega)$.

Proof. According to Lemma 5.2, $\bar{y}^{*}$ is bounded in $\operatorname{supp} \bar{u}$. Hence, the continuity of $\bar{w}$ implies that $\bar{\zeta}^{*}=\bar{y}^{*}-\bar{w}$ is also bounded in supp $\bar{u}$. Then, following [18], we get that $\bar{\zeta}^{*}$ is bounded in $\Omega$; therefore $\bar{y}^{*}$ is also bounded. Since $\bar{y}^{*}=\bar{y}$ almost everywhere in $\Omega$, we conclude that $\bar{y} \in L^{\infty}(\Omega)$. From Lemma 5.5 below, we get that $\bar{y} \in H_{0}^{1}(\Omega)$. Finally, from the state equation the $H^{-1}(\Omega)$-regularity of $\bar{u}$ follows.

Lemma 5.5. Let $y \in W_{0}^{1, p}(\Omega)$, with $p<\frac{n}{n-1}$, and $u \in \mathcal{M}(\Omega)$ such that the following linear equation is satisfied:

$$
\left\{\begin{aligned}
-\Delta y=u & \text { in } \Omega \\
y=0 & \text { on } \Gamma .
\end{aligned}\right.
$$

If $y \in L^{\infty}(\Omega)$, then $y \in H_{0}^{1}(\Omega), u \in H^{-1}(\Omega)$, and

$$
\|\nabla y\|_{L^{2}(\Omega)}^{2} \leq\|y\|_{L^{\infty}(\Omega)}\|u\|_{\mathcal{M}(\Omega)} .
$$

Proof. By convolution of $u$ with a sequence of mollifiers, we get $\left\{u_{k}\right\}_{k=1}^{\infty}$ in $L^{2}(\Omega)$ such that $u_{k} \stackrel{*}{\rightarrow} u$ in $\mathcal{M}(\Omega)$ and $\left\|u_{k}\right\|_{L^{1}(\Omega)} \leq\|u\|_{\mathcal{M}(\Omega)}$. Associated to $u_{k}$ we set $y_{k} \in H_{0}^{1}(\Omega)$ as the solution to

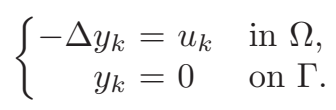

Let us take $M=\|y\|_{L^{\infty}(\Omega)}$ and define $y_{k, M}=\operatorname{proj}_{[-M,+M]}\left(y_{k}\right)$. From the equation satisfied by $y_{k}$ we deduce

$$
\begin{aligned}
\left\|\nabla y_{k, M}\right\|_{L^{2}(\Omega)}^{2} & =\int_{\Omega} \nabla y_{k} \nabla y_{k, M} d x=\int_{\Omega} u_{k} y_{k, M} d x \\
& \leq M\left\|u_{k}\right\|_{L^{1}(\Omega)} \leq\|y\|_{L^{\infty}(\Omega)}\|u\|_{\mathcal{M}(\Omega)} .
\end{aligned}
$$

First, we observe that $y_{k} \rightarrow y$ in $W_{0}^{1, p}(\Omega)$, hence $y_{k, M} \rightarrow y=\operatorname{proj}_{[-M,+M]}(y)$ in $W_{0}^{1, p}(\Omega)$ too. But the above inequality means that $\left\{y_{k, M}\right\}_{k=1}^{\infty}$ is a bounded sequence in $H_{0}^{1}(\Omega)$, hence $y$ belongs also to $H_{0}^{1}(\Omega)$ and consequently $u \in H^{-1}(\Omega)$. Moreover,

$$
\|\nabla y\|_{L^{2}(\Omega)}^{2} \leq \liminf _{k \rightarrow \infty}\left\|\nabla y_{k, M}\right\|_{L^{2}(\Omega)}^{2} \leq\|y\|_{L^{\infty}(\Omega)}\|u\|_{\mathcal{M}(\Omega)}
$$


Proof of inequality (5.2). Since $\bar{y} \in H_{0}^{1}(\Omega) \cap L^{\infty}(\Omega)$ and $\bar{u} \in H^{-1}(\Omega) \cap \mathcal{M}(\Omega)$, we can multiply (1.2), with $u=\bar{u}$ and $y=\bar{y}$, by $\bar{y}$ and using (5.5) we get

$$
\begin{aligned}
\|\nabla \bar{y}\|_{L^{2}(\Omega)}^{2} & \leq \int_{\Omega}|\nabla \bar{y}|^{2} d x+\int_{\Omega}[a(x, \bar{y}(x))-a(x, 0)] \bar{y}(x) d x \\
& =\int_{\Omega} \bar{y} d \bar{u}-\int_{\Omega} a(x, 0) \bar{y}(x) d x \\
& =\int_{\Omega} \bar{y}^{*}(x) d \bar{u}^{+}(x)-\int_{\Omega} \bar{y}^{*}(x) d \bar{u}^{-}(x)-\int_{\Omega} a(x, 0) \bar{y}(x) d x \\
& \leq\left\|y_{d}\right\|_{L^{\infty}(\Omega)}\left\|\bar{u}^{+}\right\|_{\mathcal{M}(\Omega)}+\left\|y_{d}\right\|_{L^{\infty}(\Omega)}\left\|\bar{u}^{-}\right\|_{\mathcal{M}(\Omega)}+\|a(\cdot, 0)\|_{L^{q_{1}}(\Omega)}\|\bar{y}\|_{L^{q_{1}^{\prime}}(\Omega)} \\
& \leq\left\|y_{d}\right\|_{L^{\infty}(\Omega)}\|\bar{u}\|_{\mathcal{M}(\Omega)}+C\|a(\cdot, 0)\|_{L^{q_{1}}(\Omega)}\|\nabla \bar{y}\|_{L^{2}(\Omega)},
\end{aligned}
$$

which obviously implies (5.2).

Proof of inequality (5.1). Let us prove that

$$
\bar{y}^{*}(x) \leq C\left(\left\|y_{d}\right\|_{L^{\infty}(\Omega)}+\|a(\cdot, 0)\|_{L^{q_{1}}(\Omega)}\right) \quad \forall x \in \Omega .
$$

The lower estimate is proved similarly. By (5.5), the estimate (5.6) holds in supp $\bar{u}^{+}$. From the identity $\bar{y}^{*}=\bar{w}+\bar{\zeta}^{*}$, we deduce that $\bar{\zeta}^{+*}=\bar{y}^{*}-\bar{w}+\bar{\zeta}^{-*}$. Since $\bar{\zeta}^{-*}$ is continuous in a neighborhood containing supp $\bar{u}^{+}$, and since $\bar{w}$ is continuous in $\bar{\Omega}$, and taking into account (5.5), we obtain

$$
\bar{\zeta}^{+*}(x) \leq\left\|y_{d}\right\|_{L^{\infty}(\Omega)}+\|\bar{w}\|_{L^{\infty}(\Omega)}+\left\|\bar{\zeta}^{-*}\right\|_{L^{\infty}\left(\operatorname{supp} \bar{u}^{+}\right)}<+\infty \quad \forall x \in \operatorname{supp} \bar{u}^{+} .
$$

Then, the same upper estimate for $\bar{\zeta}^{+*}$ holds in the whole domain $\Omega$; see [18]. Therefore, applying [1, Corollary 4.5.2], we obtain a sequence of compact sets $\left\{E_{k}\right\}$, with $E_{k} \subset \operatorname{supp} \bar{u}^{+}$such that

$$
\bar{u}^{+}\left(\Omega \backslash E_{k}\right)=\bar{u}^{+}\left(\operatorname{supp} \bar{u}^{+} \backslash E_{k}\right) \rightarrow 0 \text { as } k \rightarrow \infty \text { and } \zeta_{k} \in C_{0}(\Omega),
$$

where

$$
\zeta_{k}(x)=\int_{E_{k}} \mathcal{G}(x, \xi) d \bar{u}^{+}
$$

It is obvious that $\zeta_{k}(x) \leq \bar{\zeta}^{+*}(x)$ for every $x \in \Omega$ and every $k$. Therefore, $\left\{\zeta_{k}\right\}_{k}$ is a sequence of uniformly bounded functions. As a consequence of Lemma 5.5, we have that $\left\{\zeta_{k}\right\}_{k}$ is also uniformly bounded in $H_{0}^{1}(\Omega)$. We set

$$
y_{k}=\zeta_{k}+\bar{w}-\bar{\zeta}^{-*} \text {. }
$$

Hence, $y_{k} \in H_{0}^{1}(\Omega)$ and $y_{k}(x) \leq \bar{y}^{*}(x) \leq\left\|y_{d}\right\|_{L^{\infty}(\Omega)}$ for every $x \in \operatorname{supp} \bar{u}^{+}$. Since $\bar{w}$ and $\zeta_{k}$ are continuous in $\Omega$, and since $\bar{\zeta}^{-*}$ is continuous outside supp $\bar{u}^{-}$, we get that $y_{k}$ is also continuous outside $\operatorname{supp} \bar{u}^{-}$. Therefore, we have

$$
\lim _{x \rightarrow x_{0}} y_{k}(x)=y_{k}\left(x_{0}\right) \leq \bar{y}^{*}\left(x_{0}\right) \leq\left\|y_{d}\right\|_{L^{\infty}(\Omega)} \quad \forall x_{0} \in \partial\left(\Omega \backslash \operatorname{supp} \bar{u}^{+}\right) .
$$

We are going to prove that the upper estimate (5.6) is satisfied by $y_{k}$. Then, using that $y_{k}(x) \rightarrow \bar{y}^{*}(x)$ for every $x \in \Omega \backslash \operatorname{supp} \bar{u}^{+}$, we conclude (5.6). This pointwise convergence follows from (5.7):

$$
\begin{aligned}
\left|\bar{y}^{*}(x)-y_{k}(x)\right| & =\bar{\zeta}^{+*}(x)-\zeta_{k}(x)=\int_{\Omega \backslash E_{k}} \mathcal{G}(x, \xi) d \bar{u}^{+}(\xi) \\
& \leq\|\mathcal{G}(x, \cdot)\|_{C\left(\operatorname{supp} \bar{u}^{+}\right)} \bar{u}^{+}\left(\Omega \backslash E_{k}\right) \rightarrow 0 .
\end{aligned}
$$

Copyright $@$ by SIAM. Unauthorized reproduction of this article is prohibited. 
To prove the upper bound of $y_{k}$ we define for every $\eta>\left\|y_{d}\right\|_{L^{\infty}(\Omega)}$

$$
y_{k, \eta}(x)=\max \left\{0, y_{k}(x)-\eta\right\} .
$$

The rest of the proof is divided into two parts. First we prove that $y_{k, \eta} \in H_{0}^{1}(\Omega \backslash$ $\left.\operatorname{supp} \bar{u}^{+}\right)$for every $\eta>\left\|y_{d}\right\|_{L^{\infty}(\Omega)}$ and later we prove the estimate for $y_{k}$.

Proof of $y_{k, \eta} \in H_{0}^{1}\left(\Omega \backslash \operatorname{supp} \bar{u}^{+}\right)$. It is clear that $y_{k, \eta} \in H^{1}(\Omega)$ because $y_{k} \in$ $H_{0}^{1}(\Omega)$. The issue is to establish that $y_{k, \eta}$ is the limit of a sequence of functions $\left\{\psi_{j}\right\} \subset C_{0}^{\infty}\left(\Omega \backslash \operatorname{supp} \bar{u}^{+}\right)$, i.e., every $\psi_{j}$ is of class $C^{\infty}$ and has a compact support in $\Omega \backslash \operatorname{supp} \bar{u}^{+}$. First, we notice that there exists an open set $\Omega_{0} \subset \bar{\Omega}_{0} \subset\left(\Omega \backslash \operatorname{supp} \bar{u}^{+}\right)$ such that $y_{k, \eta}(x)=0$ for every $x \notin \Omega_{0}$. Indeed, if this is false, we can take a sequence $\left\{x_{j}\right\} \subset \Omega \backslash \operatorname{supp} \bar{u}^{+}$such that $x_{j} \rightarrow \hat{x}$, with $\hat{x} \in \partial\left(\Omega \backslash \operatorname{supp} \bar{u}^{+}\right)$, and $y_{k}(\hat{x})-\eta=\lim _{j \rightarrow \infty} y_{k, \eta}\left(x_{j}\right) \geq 0$. But this implies that $y_{k}(\hat{x})>\left\|y_{d}\right\|_{L^{\infty}(\Omega)}$, which contradicts (5.8). Now, making the convolution of $y_{k, \eta}$ with a sequence of mollifiers, we get functions in $C_{0}^{\infty}\left(\Omega \backslash \operatorname{supp} \bar{u}^{+}\right)$converging in $H^{1}\left(\Omega \backslash \operatorname{supp} \bar{u}^{+}\right)$to $y_{k, \eta}$. This proves that $y_{k, \eta} \in H_{0}^{1}\left(\Omega \backslash \operatorname{supp} \bar{u}^{+}\right)$.

Proof of the estimate for $y_{k}$. Observe that $\Delta y_{k}=\Delta \bar{y}$ in $\Omega \backslash \operatorname{supp} \bar{u}^{+}$and $a\left(x, y_{k}(x)\right) \leq a(x, \bar{y}(x))$ in the same set. Since $\bar{y}$ satisfies

$$
-\Delta y+a(x, y)=-\bar{u}^{-} \leq 0 \quad \text { in } \quad \Omega \backslash \operatorname{supp} \bar{u}^{+},
$$

$y_{k}$ is a subsolution of the equation

$$
-\Delta y+a(x, y)=0 \quad \text { in } \quad \Omega \backslash \operatorname{supp} \bar{u}^{+} .
$$

This can be written

$$
-\Delta y_{k}+\left[a\left(x, y_{k}\right)-a(x, 0)\right] \leq-a(x, 0) \quad \text { in } \quad \Omega \backslash \operatorname{supp} \bar{u}^{+} .
$$

Finally, we can proceed as in [21, Proof of Theorem 4.1], taking into account that the test function $y_{k, \eta}$ belongs to $H_{0}^{1}\left(\Omega \backslash \operatorname{supp} \bar{u}^{+}\right)$and the nonlinear term $\left[a\left(x, y_{k}\right)-a(x, 0)\right]$ can be removed using the fact that $\left[a\left(x, y_{k}\right)-a(x, 0)\right] y_{k, \eta} \geq 0$. Thus we get the desired estimate.

Remark 5.6. (i) Let us assume that (A1) holds with $\phi_{0} \in L^{q}(\Omega)$ for some $q>\frac{n}{2}$ and $r<3$. Given $u \in \mathcal{M}(\Omega)$, we consider (2.4) and (2.6) so that the solution $y$ to (1.2) can be written $y=w+\zeta$. Then, $y$ is in $L^{\infty}(\Omega)$ if and only if $\zeta \in L^{\infty}(\Omega)$. Indeed, if $\zeta \in L^{\infty}(\Omega)$, then $a(\cdot, \zeta) \in L^{q}(\Omega)$, and therefore (2.6) implies that $w \in L^{\infty}(\Omega)$ too; consequently $y \in L^{\infty}(\Omega)$. Conversely, if $y \in L^{\infty}(\Omega)$, then $a(\cdot, y) \in L^{q}(\Omega)$, and from the equation

$$
\left\{\begin{aligned}
-\Delta w+a(x, y)=0 & \text { in } \Omega \\
w=0 & \text { on } \Gamma
\end{aligned}\right.
$$

we get that $w \in L^{\infty}(\Omega)$, which implies that $\zeta \in L^{\infty}(\Omega)$.

This equivalence implies that the set of measures $u \in \mathcal{M}(\Omega)$ leading to solutions of (1.2) belonging to $L^{\infty}(\Omega)$ is vector space, which we denote by

$$
\mathcal{M}_{\infty}(\Omega)=\left\{u \in \mathcal{M}(\Omega) \text { : the solution to (2.4) belongs to } L^{\infty}(\Omega)\right\} .
$$

(ii) Let us observe that the more strict assumption $r<2$ was assumed in Theorem 5.1. The reason for that was to guarantee that either $\bar{\zeta}$ or $\bar{y}$ are in $L^{\infty}(\Omega)$. 
(iii) If $u \in \mathcal{M}_{\infty}(\Omega)$ and $\operatorname{supp} u^{+}$and $\operatorname{supp} u^{-}$are disjoint, then $|\bar{u}|, u^{+}, u^{-} \in$ $\mathcal{M}_{\infty}(\Omega)$ as well. To prove this we consider the decomposition $\zeta^{*}=\zeta^{+*}-\zeta^{-*}$ introduced in the proof of Theorem 5.1. Then, $\zeta^{-*}$ is continuous in a neighborhood of the support of $\bar{u}^{+}$and hence is bounded. Consequently, $0 \leq \zeta^{+*}=\zeta^{*}+\zeta^{-*}$ is bounded from above in such a neighborhood. Using again [18] we deduce that $\zeta^{+*}$ is bounded. Analogously, we prove the boundedness of $\zeta^{-*}$. Thus the solution $\zeta^{+*}+\zeta^{-*}$ to $(2.4)$ corresponding to the measure $|\bar{u}|$ is bounded, which implies that $|\bar{u}| \in \mathcal{M}_{\infty}(\Omega)$. Consequently, $\bar{u}^{+}=\frac{1}{2}(|\bar{u}|+\bar{u})$ and $\bar{u}^{-}=\frac{1}{2}(|\bar{u}|-\bar{u})$ also belong to $\mathcal{M}_{\infty}(\Omega)$.

Remark 5.7. Let us address the situation in which the observation $y_{d}$ is supported in a open subset $\mathcal{O} \subset \Omega$. In this situation, all the previous results in sections 3 and 4 remain valid with the obvious modifications. In particular the adjoint state equation must be written as follows:

$$
\left\{\begin{aligned}
-\Delta \varphi+\frac{\partial a}{\partial y}(x, y) \varphi & =\left(y-y_{d}\right) \chi_{\mathcal{O}} & & \text { in } \Omega, \\
\varphi & =0 & & \text { on } \Gamma,
\end{aligned}\right.
$$

where $\chi_{\mathcal{O}}$ denotes the characteristic function of $\mathcal{O}$.

Theorem 5.1 is also valid if we introduce an additional assumption. Namely, we assume that $\Omega \backslash \overline{\mathcal{O}}$ consists of finitely many components $\left\{\Omega_{i}\right\}_{i=1}^{m}$ satisfying $\partial \Omega_{i} \cap \Gamma \neq \emptyset$ for every $i=1, \ldots, m$. Indeed, according to the location of the point $x^{0}$ selected in the proof of Lemma 5.2, we distinguish two cases. First, let $x^{0} \in \overline{\mathcal{O}}$; then the equation in the ball $B_{\rho}\left(x^{0}\right)$ is written

$$
-\Delta \bar{\varphi}+\frac{\partial a}{\partial y}(x, \bar{y}) \bar{\varphi}=\left(y^{*}-y_{d}\right) \chi_{\mathcal{O}} \geq 0 \quad \text { in } \quad B_{\rho}\left(x^{0}\right)
$$

with $\left(y^{*}-y_{d}\right) \chi_{\mathcal{O}} \not \equiv 0$. Then, the strong maximum principle can be applied as before. Second, we assume that $x^{0} \in \Omega_{i}$ for some $i$. Then we have

$$
-\Delta \bar{\varphi}+\frac{\partial a}{\partial y}(x, \bar{y}) \bar{\varphi}=0 \quad \text { in } \Omega_{i} .
$$

Since $\|\bar{\varphi}\|_{C_{0}(\Omega)}=\alpha$ and $\bar{\varphi}\left(x^{0}\right)=-\alpha$, the maximum principle implies that $\bar{\varphi} \equiv-\alpha$ in $\Omega_{i}$, which contradicts the fact that $\bar{\varphi}=0$ on $\Gamma \cap \partial \Omega_{i}$.

In the case there exists a connected component $\Omega_{i}$ such that $\Gamma \cap \partial \Omega_{i}=\emptyset$, we assume that $\left\{x \in \Omega_{i}: \frac{\partial a}{\partial y}(x, s)>0\right.$ for all $\left.s \in \mathbb{R}\right\}$ has positive Lebesgue measure. In this case the only constant function satisfying the last equation is the zero function, which is a contradiction with the fact that $\bar{\varphi}\left(x^{0}\right)=-\alpha$.

6. Dealing with highly nonlinear terms $\boldsymbol{a}(\boldsymbol{x}, \boldsymbol{y})$. Remark 5.6 suggests the possibility to formulate the control problem $\left(\mathrm{P}_{\infty}\right)$ for highly nonlinear functions $a(x, y)$ under the more restrictive assumption on the control: $u \in \mathcal{M}_{\infty}(\Omega)$. Hereafter, $\left(\mathrm{P}_{\infty}\right)$ will denote the control problem

$$
\left(\mathrm{P}_{\infty}\right) \quad \min _{u \in \mathcal{M}_{\infty}(\Omega)} J(u)=\frac{1}{2}\left\|y-y_{d}\right\|_{L^{2}(\Omega)}^{2}+\alpha\|u\|_{\mathcal{M}(\Omega)},
$$

where $y$ is the unique solution of (1.2). In what follows we will assume that $y_{d} \in$ $L^{\infty}(\Omega)$. The following hypotheses are assumed for the function $a$.

(A4) The mapping $a: \Omega \times \mathbb{R} \rightarrow \mathbb{R}$ is a Carathéodory function of class $C^{1}$ with respect to the second variable for almost all $x \in \Omega$, and it satisfies for almost all $x \in \Omega$ and all $s \in \mathbb{R}$

$$
\left\{\begin{array}{l}
a(\cdot, 0) \in L^{q_{1}}(\Omega) \text { for some } q_{1}>\frac{n}{2}, \quad \text { and } \quad \forall M>0 \exists C_{M}>0 \text { such that } \\
0 \leq \partial_{y} a(x, s) \leq \phi_{1}(x)+C_{M} \quad \forall|s| \leq M \quad \text { and a.a. } \quad x \in \Omega \text { with } \phi_{1} \in L^{q_{1}}(\Omega)
\end{array}\right.
$$


Let us prove that $\left(\mathrm{P}_{\infty}\right)$ is well formulated, which means that $y$ is uniquely defined for every $u \in \mathcal{M}_{\infty}(\Omega)$.

ThEOREM 6.1. Under assumption (A4), there exists for every $u \in \mathcal{M}_{\infty}(\Omega)$ a unique solution $y \in H_{0}^{1}(\Omega) \cap L^{\infty}(\Omega)$ of $(1.2)$.

Proof. To prove existence we proceed as in the proof of Theorem 2.1 and consider the Dirichlet problems (2.4) and (2.6). By the definition of $\mathcal{M}_{\infty}(\Omega)$ and Lemma 5.5, we have that $\zeta \in H_{0}^{1}(\Omega) \cap L^{\infty}(\Omega)$. Hence, assumption (A4) implies that $f \in L^{q}(\Omega)$ with $q>\frac{n}{2}$. Therefore, (2.6) has a unique solution $w \in H_{0}^{1}(\Omega) \cap L^{\infty}(\Omega)$. Thus, we get that $y=\zeta+w \in H_{0}^{1}(\Omega) \cap L^{\infty}(\Omega)$ is a solution of (1.2). To prove the uniqueness, we first observe that Lemma 5.5 implies that $\mathcal{M}_{\infty}(\Omega) \subset H^{-1}(\Omega)$. Now, if $y_{1}, y_{2} \in H_{0}^{1}(\Omega) \cap L^{\infty}(\Omega)$ satisfy (1.2), then we multiply the corresponding equations by $y_{2}-y_{1}$ and subtracting we get

$$
\int_{\Omega}\left|\nabla y_{2}-\nabla y_{1}\right|^{2} d x+\int_{\Omega}\left[a\left(x, y_{2}(x)\right)-a\left(x, y_{1}(x)\right)\right]\left(y_{2}(x)-y_{1}(x)\right) d x=0 .
$$

Monotonicity of $a$ with respect to the second component implies that $y_{2}=y_{1}$.

The difficult issue is to prove that $\left(\mathrm{P}_{\infty}\right)$ has at least one solution. To this end, given $M>0$, we consider a function $\gamma_{M}: \mathbb{R} \rightarrow[-M-1,+M+1]$ of class $C^{2}$ having the properties

$$
\gamma_{M}(t)=\left\{\begin{array}{cl}
M+1 & \text { if } t>M+1, \\
t & \text { if }|t| \leq M, \\
-M-1 & \text { if } t<-M-1,
\end{array} \quad \text { and }\left|\gamma_{M}^{\prime}(t)\right|+\left|\gamma_{M}^{\prime \prime}(t)\right| \leq C_{\gamma} \forall t \in \mathbb{R},\right.
$$

for some constant $C_{\gamma}$ independent of $M$. For instance, we can select

$$
\gamma_{M}(t)=\left\{\begin{array}{cl}
M+1 & \text { if } t>M+1, \\
p(t-M)+M & \text { if } M \leq t \leq M+1, \\
t & \text { if }|t| \leq M, \\
p(t+M+1)-M-1 & \text { if }-M-1 \leq t \leq-M, \\
-M-1 & \text { if } t<-M-1,
\end{array}\right.
$$

where $p(t)=3 t^{5}-7 t^{4}+4 t^{3}+t$. With this choice, we can take $C_{\gamma}=\left\|p^{\prime}\right\|_{C[0,1]}+$ $\left\|p^{\prime \prime}\right\|_{C([0,1]}$.

Now, we define $a_{M}: \Omega \times \mathbb{R} \rightarrow \mathbb{R}$ by $a_{M}(x, t)=a\left(x, \gamma_{M}(t)\right)$. Hence, by assumption (A4), $a_{M}$ is of class $C^{1}$ with respect to the second variable and, using the mean value theorem, we get for every $s \in \mathbb{R}$ and almost all $x \in \Omega$

$$
\begin{aligned}
& 0 \leq \partial_{y} a_{M}(x, s) \leq\left(\phi_{1}(x)+C_{M+1}\right) C_{\gamma} \text { and } \\
& \left|a_{M}(x, s)\right| \leq\left[|a(x, 0)|+\phi_{1}(x) C_{\gamma}(M+1)\right]+C_{M+1} C_{\gamma}(M+1) .
\end{aligned}
$$

Now, we define the control problems

$$
\left(\mathrm{P}_{M}\right) \quad \min _{u \in \mathcal{M}(\Omega)} J_{M}(u)=\frac{1}{2}\left\|y-y_{d}\right\|_{L^{2}(\Omega)}^{2}+\alpha\|u\|_{\mathcal{M}(\Omega)},
$$

where $y$ is the solution of the state equation

$$
\left\{\begin{aligned}
-\Delta y+a_{M}(x, y) & =u & & \text { in } \Omega \\
y & =0 & & \text { on } \Gamma .
\end{aligned}\right.
$$

Copyright $@$ ㅇ by SIAM. Unauthorized reproduction of this article is prohibited. 
Since $a_{M}$ satisfies assumptions (A1) and (A2), the existence of a global minimum $u_{M}$ for $\left(\mathrm{P}_{M}\right)$, with associated state $y_{M}$, is immediate. Moreover, $\left(u_{M}, y_{M}\right)$ along with the adjoint state $\varphi_{M}$ satisfy the optimality system (3.5)-(3.7), which we write

$$
\begin{gathered}
\left\{\begin{array}{cl}
-\Delta \varphi_{M}+\frac{\partial a_{M}}{\partial y}\left(x, y_{M}\right) \varphi_{M}=y_{M}-y_{d} & \text { in } \Omega, \\
\varphi_{M}=0 & \text { on } \Gamma,
\end{array}\right. \\
\alpha\left\|u_{M}\right\|_{\mathcal{M}(\Omega)}+\int_{\Omega} \varphi_{M} d u_{M}=0, \\
\left\|\varphi_{M}\right\|_{C_{0}(\Omega)} \begin{cases}=\alpha & \text { if } u_{M} \neq 0, \\
\leq \alpha & \text { if } u_{M}=0 .\end{cases}
\end{gathered}
$$

Moreover, if $u_{M} \neq 0$, then

$$
\begin{aligned}
& \operatorname{supp}\left(u_{M}^{+}\right) \subset\left\{x \in \Omega: \varphi_{M}(x)=-\alpha\right\}, \\
& \operatorname{supp}\left(u_{M}^{-}\right) \subset\left\{x \in \Omega: \varphi_{M}(x)=+\alpha\right\} .
\end{aligned}
$$

Let us prove that $u_{M} \in \mathcal{M}_{\infty}(\Omega)$ and it is a solution of $\left(\mathrm{P}_{\infty}\right)$ for $M$ sufficiently large.

Theorem 6.2. Suppose that (A4) holds. Then there exists $M_{0}>0$ such that $u_{M}$ is a solution of $\left(\mathrm{P}_{\infty}\right)$ for every $M \geq M_{0}$.

Proof. First, we observe that Theorem 5.1 implies $u_{M} \in H^{-1}(\Omega) \cap \mathcal{M}(\Omega)$ and $y_{M} \in H_{0}^{1}(\Omega) \cap L^{\infty}(\Omega)$. Now, from Remark 5.6(i) it follows that $u_{M} \in \mathcal{M}_{\infty}(\Omega)$. Moreover (5.1) leads to

$$
\left\|y_{M}\right\|_{L^{\infty}(\Omega)} \leq C\left(\left\|y_{d}\right\|_{L^{\infty}(\Omega)}+\|a(\cdot, 0)\|_{L^{q_{1}}(\Omega)}\right) .
$$

Set $M_{0}$ equal to the right-hand side of the above inequality. Then, we have that $\left\|y_{M}\right\|_{L^{\infty}(\Omega)} \leq M_{0}$ for all $M>0$. As a consequence we get that $a_{M}\left(x, y_{M}\right)=a\left(x, y_{M}\right)$ for all $M \geq M_{0}$.

Let us prove the boundedness of $\left\{u_{M}\right\}_{M>0}$ and $\left\{y_{M}\right\}_{M>0}$ in $\mathcal{M}(\Omega)$ and $H_{0}^{1}(\Omega)$, respectively. From the definition of $J_{M}$ and the optimality of $u_{M}$ we obtain

$$
\alpha\left\|u_{M}\right\|_{\mathcal{M}(\Omega)} \leq J_{M}\left(u_{M}\right) \leq J_{M}(0)=J(0) \quad \forall M>0 .
$$

Hence, $\left\|u_{M}\right\|_{\mathcal{M}(\Omega)} \leq J(0) / \alpha$ for every $M>0$. Now, from (5.2) it follows

$$
\left\|y_{M}\right\|_{H_{0}^{1}(\Omega)} \leq C\left(\left\|y_{d}\right\|_{L^{\infty}(\Omega)} \frac{J(0)}{\alpha}+\|a(\cdot, 0)\|_{L^{q_{1}}(\Omega)}^{2}\right)^{1 / 2} .
$$

Let us take a subsequence of $\left\{\left(u_{M}, y_{M}\right)\right\}_{M \geq M_{0}}$, denoted in the same form, such that

$$
u_{M} \stackrel{*}{\rightarrow} \bar{u} \text { in } \mathcal{M}(\Omega), \quad u_{M} \rightarrow \bar{u} \text { in } H^{-1}(\Omega), \quad y_{M} \rightarrow \bar{y} \text { in } H_{0}^{1}(\Omega) .
$$

We have that $\bar{y}$ also satisfies (5.1) and (5.2), that it is the state associated to $\bar{u}$, and therefore $\bar{u} \in \mathcal{M}_{\infty}(\Omega)$. Moreover, for every $u \in \mathcal{M}_{\infty}(\Omega)$

$$
J(\bar{u}) \leq \liminf _{M \rightarrow \infty} J\left(u_{M}\right)=\liminf _{M \rightarrow \infty} J_{M}\left(u_{M}\right) \leq \liminf _{M \rightarrow \infty} J_{M}(u)=J(u) .
$$

Copyright $($ C by SIAM. Unauthorized reproduction of this article is prohibited. 
Hence, $\bar{u}$ is a solution of $\left(\mathrm{P}_{\infty}\right)$. But, we also have for every $M \geq M_{0}$

$$
J(\bar{u}) \leq J\left(u_{M}\right)=J_{M}\left(u_{M}\right) \leq J_{M}(\bar{u})=J(\bar{u}),
$$

where we used that $u_{M}$ is a solution of $\left(\mathrm{P}_{M}\right)$. Therefore, $J\left(u_{M}\right)=J(\bar{u})$ for every $M \geq M_{0}$, and consequently $u_{M}$ is a solution of $\left(\mathrm{P}_{\infty}\right)$ for all $M \geq M_{0}$.

Next we study second order optimality conditions. To this end we make the following assumption.

(A5) The mapping $a$ is a Carathéodory function of class $C^{2}$ with respect to the second variable for almost all $x \in \Omega$, and it satisfies

$$
\left\{\begin{array}{l}
\exists \phi_{2} \in L^{q_{2}}(\Omega) \text { with } q_{2}>n \text { and } \forall M>0 \exists C_{M}>0 \text { such that } \\
\left|\partial_{y}^{2} a(x, s)\right| \leq\left|\phi_{2}(x)\right|+C_{M} \text { for a.a. } x \in \Omega \text { and } \forall|s| \leq M .
\end{array}\right.
$$

Since (A4) and (A5) replace (A1), (A2), and (A3), we cannot rely on Theorem 2.2 to deduce the differentiability of the control-to-state mapping. Consequently, we do not have the differentiability of $F$ stated in Proposition 3.1. However, let us observe that under assumptions (A4) and (A5), (1.2), (2.9), and (2.10) and expressions (3.1) and (3.3) for $F^{\prime}$ and $F^{\prime \prime}$ are well defined for every $u \in \mathcal{M}_{\infty}(\Omega)$ and $v, v_{1}, v_{2} \in \mathcal{M}(\Omega)$. If we perturb $u$ in $\mathcal{M}(\Omega)$, then the existence of a solution to these equations may fail. Since our goal is to get first and second order necessary conditions for local optimality, differentiability of $F$ is required. This motivates the introduction of a stronger norm in $\mathcal{M}_{\infty}(\Omega)$ where this differentiability holds. We define the norm

$$
\|u\|_{\mathcal{M}_{\infty}(\Omega)}=\|u\|_{\mathcal{M}(\Omega)}+\|\zeta\|_{L^{\infty}(\Omega)},
$$

where $\zeta$ is the solution to (2.4). Endowed with this norm, $\mathcal{M}_{\infty}(\Omega)$ is a Banach space. Now we introduce the space of associated states as

$$
V_{\infty}(\Omega)=\left\{y \in H_{0}^{1}(\Omega) \cap L^{\infty}(\Omega): \Delta y \in \mathcal{M}(\Omega)\right\},
$$

endowed with the norm

$$
\|y\|_{V \infty(\Omega)}=\|y\|_{H_{0}^{1}(\Omega)}+\|y\|_{L^{\infty}(\Omega)}+\|\Delta y\|_{\mathcal{M}(\Omega)} .
$$

$V_{\infty}(\Omega)$ is also a Banach space. From Theorem 6.1 we know that the mapping $G_{\infty}$ : $\mathcal{M}_{\infty}(\Omega) \rightarrow V_{\infty}(\Omega)$ is well defined. To prove the differentiability we argue as in the proof of Theorem 2.2. To this end we define $\mathcal{F}_{\infty}: V_{\infty}(\Omega) \times \mathcal{M}_{\infty}(\Omega) \rightarrow \mathcal{M}_{\infty}(\Omega)$ by

$$
\mathcal{F}_{\infty}(y, u)=-\Delta y+a(\cdot, y)-u
$$

From (A4), respectively, (A5), it follows that the mapping $y \rightarrow a(\cdot, y)$ is $C^{1}$, respectively, $C^{2}$, from $V_{\infty}(\Omega)$ to $L^{q_{1}}(\Omega) \subset \mathcal{M}_{\infty}(\Omega)$. Then following the same arguments as in the proof of Theorem 2.2, we obtain that $G_{\infty}$ is $C^{1}$, respectively, $C^{2}$, and that (2.9) and (2.10) hold. Furthermore, by using the chain rule, we deduce the differentiability of $F: \mathcal{M}_{\infty}(\Omega) \rightarrow \mathbb{R}$ and the identities (3.1) and (3.3) for every $v, v_{1}, v_{2} \in \mathcal{M}_{\infty}(\Omega)$. It is immediate from the expressions (3.1) and (3.3) that the linear and bilinear forms $F^{\prime}(u)$ and $F^{\prime \prime}(u)$ can be extended to linear and bilinear continuous forms on $\mathcal{M}(\Omega)$ and $\mathcal{M}(\Omega) \times \mathcal{M}(\Omega)$ for every $u \in \mathcal{M}_{\infty}(\Omega)$. These extensions are unique by the weak* density of $\mathcal{M}_{\infty}(\Omega)$ in $\mathcal{M}(\Omega)$. 
To formulate the second order necessary optimality conditions we define the analogous cone to $(3.9)$

$$
C_{\infty, \bar{u}}=\left\{v \in \mathcal{M}_{\infty}(\Omega): F^{\prime}(\bar{u}) v+\alpha j^{\prime}(\bar{u} ; v)=0\right\},
$$

where $\bar{u} \in \mathcal{M}_{\infty}(\Omega)$ satisfies the first order optimality conditions.

THEOREM 6.3. Under assumption (A4), if $\bar{u}$ is a local solution of $\left(\mathrm{P}_{\infty}\right)$, then there exists $\bar{\varphi} \in W_{0}^{1, p^{\prime}}(\Omega)$ for some $p^{\prime}>n$ such that (3.5)-(3.7) hold. If in addition (A5) is satisfied, then $F^{\prime \prime}(\bar{u}) v^{2} \geq 0$ for every $v \in C_{\infty, \bar{u}}$.

Proof. The first order necessary optimality conditions can be proved as in Theorem 3.4. In the proof of second order necessary conditions given in Theorem 3.7 , the only issue to take into account is the following one. For $v \in \mathcal{M}_{\infty}(\Omega)$, we construct $v_{k}$ in the same way, but we have to prove that $v_{k} \in \mathcal{M}_{\infty}(\Omega)$; otherwise the existence of the state associated to $\bar{u}+\rho v_{k}$ can fail. To this end, we first observe, as pointed out in Remark 5.6, that $|v| \in \mathcal{M}_{\infty}(\Omega)$ as well. Comparing the expressions

$$
|v|=\left|g_{v}\right| d|\bar{u}|+d\left|v_{s}\right| \text { and }\left|v_{k}\right|=\left|g_{v_{k}}\right| d|\bar{u}|+d\left|v_{s}\right|
$$

we obtain by the definition of $g_{v_{k}}$ that $\left|v_{k}\right| \leq|v|$. Hence, the solutions $\zeta$ and $\zeta_{k}$ of (2.4) corresponding to $|v|$ and $\left|v_{k}\right|$, respectively, satisfy $0 \leq \zeta_{k} \leq \zeta$. Since, $|v| \in \mathcal{M}_{\infty}(\Omega)$, then $\zeta \in L^{\infty}$, hence $\zeta_{k} \in L^{\infty}(\Omega)$ too. This implies that $\left|v_{k}\right| \in \mathcal{M}_{\infty}(\Omega)$. Finally, since $0 \leq v_{k}^{+} \leq\left|v_{k}\right|$ and $0 \leq v_{k}^{-} \leq\left|v_{k}\right|$, arguing in the same way we conclude that $v_{k}=v_{k}^{+}-v_{k}^{-} \in \mathcal{M}_{\infty}(\Omega)$.

Analogously to section 4 , for the sufficient conditions we introduce the extended cone

$$
C_{\infty, \bar{u}}^{\tau}=\left\{v \in \mathcal{M}_{\infty}(\Omega): F^{\prime}(\bar{u}) v+\alpha j^{\prime}(\bar{u} ; v) \leq \tau\left\|z_{v}\right\|_{L^{2}(\Omega)}\right\} .
$$

Let us denote for $M>0$

$$
B_{\infty, M}=\left\{u \in \mathcal{M}_{\infty}(\Omega) \text { : the solution } \zeta \text { to }(2.4) \text { satisfies }\|\zeta\|_{L^{\infty}(\Omega)} \leq M\right\} .
$$

Based on the cone $C_{\infty, \bar{u}}^{\tau}$ we define the second order sufficient condition:

(SOSC) There exist positive constants $\kappa, \rho, \tau$, and $M>M_{0}$ such that

$$
F^{\prime \prime}(u) v^{2} \geq \kappa\left\|z_{v}\right\|_{L^{2}(\Omega)}^{2} \quad \forall v \in C_{\infty, \bar{u}}^{\tau}, \forall u \in B_{\infty, M} \text { with }\|u-\bar{u}\|_{W^{-1, \bar{p}}(\Omega)}<\rho,
$$

where $M_{0}$ was introduced in Theorem 6.2 and $1 \leq \bar{p}<n /(n-1)$ is chosen so that $\Delta: W_{0}^{1, \bar{p}}(\Omega) \rightarrow W^{-1, \bar{p}}(\Omega)$ is an isomorphism.

THEOREM 6.4. Let $\bar{u} \in \mathcal{M}_{\infty}(\Omega)$ satisfy the first order conditions (3.5)-(3.7) and (SOSC). Under the assumptions (A4) and (A5), there exist positive constants $\varepsilon_{M} \leq \rho$ and $\sigma_{M}$ such that

$$
J(\bar{u})+\frac{\hat{\sigma}_{M}}{2}\|y(u)-\bar{y}\|_{L^{2}(\Omega)}^{2} \leq J(u) \forall u \in B_{\infty, M} \text { with }\|u-\bar{u}\|_{W^{-1, \bar{p}}(\Omega)}<\varepsilon_{M} .
$$

Proof. The proof of this theorem follows the steps of the proof of Theorem 4.2 with the following differences. First we observe that there exists a constant $K_{M}$ such that

$$
\|y(u)\|_{L^{\infty}(\Omega)} \leq K_{M} \quad \forall u \in B_{\infty, M} .
$$

Indeed, we can decompose $y(u)=\zeta+w$ with $\zeta$ solving (2.4) and $w$ satisfying (2.6). By definition of $B_{\infty, M}$ we know that $\|\zeta\|_{L^{\infty}(\Omega)} \leq M$. Moreover, (2.6) and assumption 
(A4) imply that $\|w\|_{L^{\infty}(\Omega)}$ is bounded by a constant depending on $M$, which leads to the above estimate.

In the statement of Lemma $4.3, u$ and $\bar{u}$ must belong to $B_{\infty, M}$ and $v \in B_{\infty, 2 M}$. Recall that when Lemma 4.3 is used in the proof of Theorem $4.2 v=u_{k}-\bar{u}$ with $u_{k}$ and $\bar{u}$ both belonging to $B_{\infty, M}$ in the present case. Now, the estimate in (4.11) can be obtained as follows:

$$
\begin{aligned}
\left\|\frac{\partial^{2} a}{\partial y^{2}}(x, \hat{y})(y-\bar{y})\right\|_{L^{2}(\Omega)} & \leq \sqrt{2 K_{M}}\left(\int_{\Omega}\left(\frac{\partial^{2} a}{\partial y^{2}}(x, \hat{y})\right)^{2}|y-\bar{y}| d x\right)^{1 / 2} \\
& \leq \sqrt{2 K_{M}}\left\|\frac{\partial^{2} a}{\partial y^{2}}(x, \hat{y})\right\|_{L^{q_{2}(\Omega)}}\|y-\bar{y}\|_{L^{q_{2} /\left(q_{2}-2\right)}}^{1 / 2} \\
& \leq C\left(\sqrt{2 K_{M}}\right)^{\frac{q_{2}}{q_{2}-2}}\left\|\frac{\partial^{2} a}{\partial y^{2}}(x, \hat{y})\right\|_{L^{q_{2}(\Omega)}}\|y-\bar{y}\|_{L^{1}(\Omega)}^{\frac{q_{2}-2}{2 q_{2}}} \\
& \leq C\left(\sqrt{2 K_{M}}\right)^{\frac{q_{2}}{q_{2}-2}}\left\|\frac{\partial^{2} a}{\partial y^{2}}(x, \hat{y})\right\|_{L^{q_{2}(\Omega)}}\|y-\bar{y}\|_{W_{0}^{1, \bar{p}}(\Omega)}^{\frac{q_{2}-2}{2 q_{2}}} \\
& \leq C\left(\sqrt{2 K_{M}}\right)^{\frac{q_{2}}{q_{2}-2}}\left\|\frac{\partial^{2} a}{\partial y^{2}}(x, \hat{y})\right\|_{L^{q_{2}(\Omega)}}\|u-\bar{u}\|_{W^{-1, \bar{p}}(\Omega)}^{\frac{q_{2}-2}{2 q_{2}}} .
\end{aligned}
$$

Finally, it is enough to observe that $|\hat{y}(x)| \leq K_{M}$ and to recall assumption (A5) to deduce

$$
\left|\frac{\partial^{2} a}{\partial y^{2}}(x, \hat{y})\right| \leq \phi_{2}(x)+C_{K_{M}} \quad \text { with } \phi_{2} \in L^{q_{2}}(\Omega)
$$

Similar arguments are used to get the second estimate of (4.7), taking into account that the solution of (4.8) is bounded due to the fact $v \in B_{\infty, 2 M}$.

Remark 6.5. The stability results stated in Theorems 4.6 and 4.7 remain valid for problem $\left(\mathrm{P}_{\infty}\right)$ if $\left\{y_{d, \delta}\right\}_{\delta>0}$ is uniformly bounded in $L^{\infty}(\Omega)$. For the proof we only need to take into account that the local minima $u_{\delta}$ have associated states $y_{\delta}$ uniformly bounded in $L^{\infty}(\Omega)$ by a constant

$$
\hat{M}=C\left(\max _{\delta>0}\left\|y_{d, \delta}\right\|_{L^{\infty}(\Omega)} \frac{J(0)}{\alpha}+\|a(\cdot, 0)\|_{L^{q_{1}}(\Omega)}^{2}\right)^{1 / 2}
$$

see Theorem 5.1. Then $M$ in (6.10) has to be taken so that $M \geq \max \left\{M_{0}, \hat{M}\right\}$.

\section{REFERENCES}

[1] D. Armitage and S. Gardiner, Classical Potential Theory, Springer-Verlag, Berlin, 2001.

[2] P. Benilan and H. Brezis, Nonlinear problems related to the Thomas-Fermi equation, dedicated to Philippe Benilan, J. Evol. Equ., 3 (2004), pp. 673-770.

[3] L. Boccardo and T. Gallouët, Nonlinear elliptic and parabolic equations involving measure data, J. Funct. Anal., 87 (1989), pp. 149-169.

[4] F. Bonnans and A. Shapiro, Perturbation Analysis of Optimization Problems, SpringerVerlag, Berlin, 2000.

[5] H. Brezis, M. Marcus, And A. C. Ponce, Nonlinear elliptic equations with measures revisited, in Mathematical Aspects of Nonlinear Dispersive Equations, Ann. of Math. Stud. 163, Princeton University Press, Princeton, NJ, 2007, pp. 55-109.

[6] H. Brezis And W. Strauss, Semilinear second order elliptic equations in $l^{1}$, J. Math., Soc. Japan, 4 (1973), pp. 565-589. 
[7] E. Casas, C. Clason, and K. Kunisch, Approximation of elliptic control problems in measure spaces with sparse solutions, SIAM J. Control Optim., 50 (2012), pp. 1735-1752.

[8] E. Casas, C. Clason, and K. Kunisch, Parabolic control problems in measure spaces with sparse solutions, SIAM J. Control Optim., 51 (2013), pp. 28-63.

[9] E. Casas, R. Herzog, and G. Wachsmuth, Approximation of sparse controls in semilinear equations by piecewise linear functions, Numer. Math., 122 (2012), pp. 645-669.

[10] E. Casas, R. Herzog, and G. Wachsmuth, Optimality conditions and error analysis of semilinear elliptic control problems with $L^{1}$ cost functional, SIAM J. Optim., 22 (2012), pp. 795-820.

[11] E. Casas And F. TröLtzsch, Second-order and stability analysis for state-constrained elliptic optimal control problems with sparse controls, to appear.

[12] E. Casas and E. Zuazua, Spike controls for elliptic and parabolic PDE, Systems Control Lett., 62 (2013), pp. 311-318.

[13] F. Clarke, Optimization and Nonsmooth Analysis, John Wiley \& Sons, Toronto, 1983.

[14] C. Clason and K. Kunisch, A duality-based approach to elliptic control problems in nonreflexive Banach spaces, ESAIM Control Optim. Calc. Var., 17 (2011), pp. 243-266.

[15] D. Gilbarg and N. Trudinger, Elliptic Partial Differential Equations of Second Order, Springer-Verlag, Berlin, 1977.

[16] R. Herzog, G. Stadler, and G. Wachsmuth, Directional sparsity in optimal control of partial differential equations, SIAM J. Control Optim., 50 (2012), pp. 943-963.

[17] D. Jerison and C. E. Kenig, The inhomogeneous Dirichlet problem in Lipschitz domains, J. Funct. Anal., 130 (1995), pp. 161-219.

[18] K. Pieper And B. Vexler, A priori error analysis for discretization of sparse elliptic optimal control problems in measure space, SIAM J. Control Optim., 51 (2013), pp. 2788-2808.

[19] W. Rudin, Real and Complex Analysis, McGraw-Hill, London, 1970.

[20] G. Stadler, Elliptic optimal control problems with $L^{1}$-control cost and applications for the placement of control devices, Comput. Optim. Appl., 44 (2009), pp. 159-181.

[21] G. Stampacchia, Le problème de Dirichlet pour les équations elliptiques du second ordre à coefficients discontinus, Ann. Inst. Fourier (Grenoble), 15 (1965), pp. 189-258.

[22] F. Tröltzsch, Optimal Control of Partial Differential Equations, Grad. Stud. Math., AMS, Providence, RI, 2010.

[23] G. Wachsmuth And D. Wachsmuth, Convergence and regularisation results for optimal control problems with sparsity functional, ESAIM Control Optim. Calc. Var., 17 (2011), pp. $858-886$.

Copyright $@$ ( ) by SIAM. Unauthorized reproduction of this article is prohibited. 